\title{
The stochastic tamed MHD equations: existence, uniqueness and invariant measures
}

\author{
Andre Schenke ${ }^{1}$ \\ Received: 1 September 2020 / Revised: 29 April 2021 / Accepted: 23 June 2021 / \\ Published online: 14 July 2021 \\ (c) The Author(s) 2021
}

\begin{abstract}
We study the tamed magnetohydrodynamics equations, introduced recently in a paper by the author, perturbed by multiplicative Wiener noise of transport type on the whole space $\mathbb{R}^{3}$ and on the torus $\mathbb{T}^{3}$. In a first step, existence of a unique strong solution are established by constructing a weak solution, proving that pathwise uniqueness holds and using the Yamada-Watanabe theorem. We then study the associated Markov semigroup and prove that it has the Feller property. Finally, existence of an invariant measure of the equation is shown for the case of the torus.
\end{abstract}

Keywords Tamed MHD equations - Magnetohydrodynamics - MHD equations · Porous media $\cdot$ Invariant measure $\cdot$ Strong solutions $\cdot$ Feller semigroup

Mathematics Subject Classification 76W05 - 76S05 · 35K91 · 76D03 - 60H15

\section{Introduction}

In this paper, we consider a randomly perturbed version of the tamed MHD (TMHD) equations introduced recently in [42]. This aims at modelling the turbulent behaviour of a flow of electrically conducting fluids through porous media. To be precise, we study existence and uniqueness of strong solutions, as well as existence of invariant

Andre Schenke

aschenke@math.uni-bielefeld.de

1 Fakultät für Mathematik, Universität Bielefeld, 33615 Bielefeld, Germany 
measures of the following system of equations:

$$
\left\{\begin{aligned}
\mathrm{d} \boldsymbol{v}= & {\left[\Delta \boldsymbol{v}-(\boldsymbol{v} \cdot \nabla) \boldsymbol{v}+(\boldsymbol{B} \cdot \nabla) \boldsymbol{B}+\nabla\left(p+\frac{|\boldsymbol{B}|^{2}}{2}\right)-g_{N}\left(|(\boldsymbol{v}, \boldsymbol{B})|^{2}\right) \boldsymbol{v}\right] \mathrm{d} t } \\
& +\sum_{k=1}^{\infty}\left[\left(\boldsymbol{\sigma}_{k}(t) \cdot \nabla\right) \boldsymbol{v}+\nabla p_{k}(t)+\boldsymbol{h}_{k}(t, y(t))\right] \mathrm{d} W_{t}^{k}+\boldsymbol{f}_{v}(t, y(t)) \mathrm{d} t, \\
\mathrm{~d} \boldsymbol{B} \quad & {\left[\Delta \boldsymbol{B}-(\boldsymbol{v} \cdot \nabla) \boldsymbol{B}+(\boldsymbol{B} \cdot \nabla) \boldsymbol{v}+\nabla \pi-g_{N}\left(|(\boldsymbol{v}, \boldsymbol{B})|^{2}\right) \boldsymbol{B}\right] \mathrm{d} t } \\
& +\sum_{k=1}^{\infty}\left[\left(\overline{\boldsymbol{\sigma}}_{k}(t) \cdot \nabla\right) \boldsymbol{B}+\nabla \pi_{k}(t)+\overline{\boldsymbol{h}}_{k}(t, y(t))\right] \mathrm{d} \bar{W}_{t}^{k}+\boldsymbol{f}_{B}(t, y(t)) \mathrm{d} t, \\
\operatorname{div}(\boldsymbol{v})= & 0, \quad \operatorname{div}(\boldsymbol{B})=0 .
\end{aligned}\right.
$$

Here, $\boldsymbol{v}=\boldsymbol{v}(t, x)$ denotes the velocity field of the fluid, $\boldsymbol{B}=\boldsymbol{B}(t, x)$ is the magnetic field, $p=p(t, x)$ is the pressure, $\pi=\pi(t, x)$ is the "magnetic pressure" that arises from the non-divergence-freeness of the nonlinear taming function in the equation of the magnetic field as introduced in [42, Section 1.2.3], $g_{N}$ denotes the taming function, which can be understood as an additional drag or friction term due to the porous medium. The taming function $g_{N}: \mathbb{R}_{+} \rightarrow \mathbb{R}_{+}$is defined by

$$
\begin{cases}g_{N}(r):=0, & r \in[0, N], \\ g_{N}(r):=C_{\text {taming }}\left(r-N-\frac{1}{2}\right), & r \geq N+1, \\ 0 \leq g_{N}^{\prime}(r) \leq C_{1}, & r \geq 0, \\ \left|g_{N}^{(k)}(r)\right| \leq C_{k}, & r \geq 0, k \in \mathbb{N} .\end{cases}
$$

The terms $\boldsymbol{f}_{v}=\boldsymbol{f}_{v}(t, x, y(t))$ and $\boldsymbol{f}_{B}=\boldsymbol{f}_{B}(t, x, y(t))$ are forces acting on the fluid. The form of the noise term will be discussed below. For simplicity, we have set all the constants appearing in the equations to one. For the assumptions on the forces, see Sect. 2.1.

The stochastic MHD equations were first studied by Sritharan and Sundar [44] who proved existence of martingale solutions in the two- and three-dimensional case.

Questions of ergodicity in two dimensions were studied by Barbu and Da Prato [1] for Wiener noise, and for $\alpha$-stable noise by Shen and Huang [43]. Yamazaki [51] proved ergodicity in the case of random forcing by finitely many modes. In three dimensions, he also proved ergodicity of a Faedo-Galerkin approximation of the MHD equations for degenerate noise in [50].

The asymptotic behaviour of the SMHD equations was studied in the 2D additive white noise case by Zhao and Li [54], and in the 2D fractional case by Huang and Shen [21]. Wang [46] studied the system's exponential behaviour. Furthermore, Wang and $\mathrm{Li}$ [47] proved long-time robustness of the associated random attractor.

Jump-type and fractional noises have been studied by Sundar [45]. Manna and Mohan [27], as well as Motyl [33] studied the jump noise case, and the latter author provided a nice and very general framework for 3D hydrodynamic-type equations with Lévy noise on unbounded domains, generalising the 2D framework of Chueshov and Millet [8]. Chueshov and Millet proved large deviations principles as well, and in [9] also a Wong-Zakai approximation and a support theorem.

The existence of solutions to the non-resistive MHD equations with Lévy noise was investigated by Manna et al. [28]. 
Yamazaki proved existence of global martingale solutions for the nonhomogeneous system [48]. The case of non-Newtonian electrically conducting fluids and their longtime behaviour was studied in a paper by Razafimandimby and Sango [34].

In modelling the noise, we follow the approach of Mikulevicius and Rozovskii [30-32] who proposed a multiplicative noise of transport-type for the Navier-Stokes equations, motivated by the turbulence theory of Kraichnan [23], which was further developed by Gawedzki et al. [17,18]. Transport-type noise was studied by several other authors as well, e.g. Brzeźniak et al. [4,5], as well as in Flandoli and Gatarek [13, Section 3.3, pp. 378 f.]. More recently, Hofmanová et al. [20] studied the problem via rough path methods.

We note that Mikulevicius and Rozovskii considered the case of only Hölder continuous $\sigma$ as being important to Kraichnan's turbulent velocity model, but for simplicity, we restrict ourselves to the case of differentiable $\sigma$ [see Assumption (H2) below].

The tamed MHD equations were inspired by several works on the tamed NavierStokes equations. Existence and uniqueness as well as ergodicity for the stochastic tamed Navier-Stokes equations were studied by Röckner and Zhang [40]. The study of Freidlin-Wentzell-type large deviations was carried out by Röckner et al. [38]. The case of existence, uniqueness and small time large deviation principles for the Dirichlet problem in bounded domains can be found in the work of Röckner and Zhang [37]. More recently, there has been resparked interest in the subject, with contributions by Dong and Zhang [12] (existence and uniqueness for multiplicative Lévy noise), as well as Brzeźniak and Dhariwal [3] (existence, uniqueness and existence of invariant measures in the full space $\mathbb{R}^{3}$ by different methods).

The deterministic tamed MHD equations were introduced by the author in [42], where existence, uniqueness and regularity were studied.

From a physical point of view, the fact that our model is most appropriate for low to moderate Reynolds numbers, cf. [42, Section 1.2.1], raises the question of whether or not a stochastic model for turbulence (which is commonly associated with high Reynolds numbers) is appropriate in this setting. It is, nevertheless, an interesting mathematical problem that we want to address in this work. Furthermore, the randomness can also be seen as a model for other features of our system, including uncertainty.

\subsection{Damped Navier-Stokes and MHD equations}

A related model for fluid flow through porous media is provided by the socalled (nonlinearly) damped Navier-Stokes equations, sometimes called BrinkmanForchheimer-extended Darcy Models. They are given by

$$
\frac{\partial \boldsymbol{v}}{\partial t}=\Delta \boldsymbol{v}-(\boldsymbol{v} \cdot \nabla) \boldsymbol{v}-\nabla p-\alpha|\boldsymbol{v}|^{\beta-1} \boldsymbol{v}
$$

where $\alpha>0$ and $\beta \geq 1$. The damping term $-\alpha|\boldsymbol{v}|^{\beta-1} \boldsymbol{v}$ models the resistence to the motion of the flow resulting from physical effects like porous media flow, drag or friction, or other dissipative mechanisms (cf. [7]). It represents a restoring force, which for $\beta=1$ assumes the form of classical, linear damping, whereas $\beta>1$ means 
a restoring force that grows superlinearly with the velocity (or magnetic field). Cai and Jiu [7] first proved existence and uniqueness of a global strong solution for $\frac{7}{2} \leq \beta \leq 5$.

This range was lowered down to $\beta \in(3,5]$ by Zhang et al. [53] who considered the case $\beta=3$ to be critical [53, Remark 3.1]. Zhou in [55] proved the existence of a global solution for all $\beta \in[3,5]$. For the case $\beta \in[1,3)$, he established regularity criteria that ensure smoothness. Uniqueness holds for any $\beta \geq 1$ in the class of weak solutions.

The first problems studied in the stochastic damped Navier-Stokes case were related to the inviscid limit of the damped equations for $\beta=1$ in 2D, cf. Bessaih and Ferrario [2] and Glatt-Holtz et al. [19]. You [52] proved existence of a random attractor under the assumption of well-posedness for additive noise for $\beta \in(3,5]$ (notably leaving out the critical, or tamed, case $\beta=3$ ). Yamazaki [49] proved a Lagrangian formulation and extended Kelvin's circulation theorem to the partially damped case (i.e. only a few components are damped, but the damping there is much stronger, e.g. $\beta_{k}=9$ in two components $k=3,4$ in the 4D case). Brzeźniak and Ferrario [6] showed existence of stationary solutions on the whole space $\mathbb{R}^{3}$ for $\beta=1$. Liu and Gao [24] proved existence and uniqueness of an invariant measure and a random attractor for $\beta \in[3,5]$. The same authors in [15] proved a small-time large deviation principle for the same parameter range. Furthermore, again for the same range, for multiplicative noise, exponential convergence of the weak solutions in $L^{2}$ to the stationary solution as well as stabilisation were proved by Liu et al. [25]. Finally, the case of jump noise was treated by Liu and Gao [16].

\subsection{Results and structure of the paper}

Here are the main results of the paper. In the following, the underlying domain $\mathbb{D} \subset \mathbb{R}^{3}$ is assumed to be either $\mathbb{R}^{3}$ or the torus $\mathbb{T}^{3}$. Results apply to both cases unless otherwise stated.

First, we prove existence and uniqueness of a strong solution to the stochastic tamed MHD equations.

Theorem 1.1 (Existence and uniqueness, Theorems 3.8, 3.9 and 3.10 below) Let the coefficients $f:=\left(\boldsymbol{f}_{v}, \boldsymbol{f}_{B}\right), \Sigma:=(\boldsymbol{\sigma}, \overline{\boldsymbol{\sigma}})$ and $H:=(\boldsymbol{h}, \overline{\boldsymbol{h}})$ satisfy Assumptions $(H 1)-$ (H3) and let $y_{0} \in \mathcal{H}^{1}$. Then there exists a unique strong solution y to the stochastic tamed MHD equations, in the sense of Definition 3.6, with the following properties:

(i) $y \in L^{2}\left(\Omega, P ; C\left([0, T] ; \mathcal{H}^{1}\right)\right) \cap L^{2}\left(\Omega, P ; L^{2}\left([0, T] ; \mathcal{H}^{2}\right)\right)$ for all $T>0$ and

$$
\mathbb{E}\left[\sup _{t \in[0, T]}\|y(t)\|_{\mathcal{H}^{1}}^{2}\right]+\int_{0}^{t} \mathbb{E}\left[\|y(s)\|_{\mathcal{H}^{2}}^{2}\right] \mathrm{d} s \leq C_{T, f, H}\left(1+\left\|y_{0}\right\|_{\mathcal{H}^{1}}^{2}\right) \cdot N
$$


(ii) In $\mathcal{H}^{0}$, the following equation holds:

$$
y(t)=\int_{0}^{t}[\mathcal{A}(y(s))+\mathcal{P} f(s, y(s))] \mathrm{d} s+\sum_{k=1}^{\infty} \int_{0}^{t} \mathcal{B}_{k}(s, y(s)) \mathrm{d} W_{s}^{k} \quad \forall t \geq 0, P-\text { a.s. }
$$

By the preceding theorem, for time-homogeneous coefficients, the solution process $y=\left(y\left(t ; y_{0}\right)\right)_{t \geq 0}$ is a strong Markov process. For $t \geq 0$, we can define the associated Markov semigroup on the space $B C_{\text {loc }}\left(\mathcal{H}^{1}\right)$ of bounded, locally uniformly continuous functions on the divergence-free Sobolev space $\mathcal{H}^{1}$, by

$$
T_{t} \phi\left(y_{0}\right):=\mathbb{E}\left[\phi\left(y\left(t ; y_{0}\right)\right)\right], \quad \phi \in B C_{\mathrm{loc}}\left(\mathcal{H}^{1}\right), y_{0} \in \mathcal{H}^{1} .
$$

Then, under a slightly stronger assumption on the coefficients of the noise, this semigroup satisfies the Feller property.

Theorem 1.2 (Feller property, Theorem 4.2 below) Under the Assumptions (H1), (H2) and (H3)', for every $t \geq 0, T_{t}$ maps $B C_{l o c}\left(\mathcal{H}^{1}\right)$ into itself, i.e. it is a Feller semigroup on $B C_{\text {loc }}\left(\mathcal{H}^{1}\right)$.

In the case of a periodic domain $\mathbb{D}=\mathbb{T}^{3}$, we prove the existence of an invariant measure.

Theorem 1.3 (Existence of an invariant measure, Theorem 4.3 below) Under the hypotheses (H1), (H2), (H3)', in the periodic case $\mathbb{D}=\mathbb{T}^{3}$, there exists an invariant measure $\mu \in \mathcal{P}\left(\mathcal{H}^{1}\right)$ associated to $\left(T_{t}\right)_{t \geq 0}$, i.e. a measure $\mu$ such that for every $t \geq 0, \phi \in B C_{\text {loc }}\left(\mathcal{H}^{1}\right)$

$$
\int_{\mathcal{H}^{1}} T_{t} \phi\left(y_{0}\right) \mathrm{d} \mu\left(y_{0}\right)=\int_{\mathcal{H}^{1}} \phi\left(y_{0}\right) \mathrm{d} \mu\left(y_{0}\right) .
$$

The goal of this work was to generalise the well-posedness results of Röckner and Zhang [39] for the stochastic tamed Navier-Stokes equations to the stochastic tamed MHD case. In doing so, we have to establish MHD versions of several tools they use, which is technically more difficult.

To the best of the author's knowledge, neither the stochastic damped MHD equations nor the stochastic tamed MHD equations have been considered so far. This work is a first step in this direction.

The paper is organised as follows: we state our assumptions in Sect. 2.1 as well as auxiliary results and estimates on the coefficients of our equation in Sect. 2.2. Since our proof of existence involves a tightness argument, we provide a tightness criterion in Sect. 2.3. Existence and uniqueness of a strong solution is then proved in Sect. 3. We start by defining weak and strong solutions in 3.1. The next Section 3.2 is then devoted to proving pathwise uniqueness. Existence of a weak solution is proved in Sect. 3.3 via the by now classical strategy of proving a priori estimates for the Faedo-Galerkin approximation to the equation and using them to infer tightness of the sequence of laws, Skorokhod coupling to obtain almost sure convergence, and concluding by proving 
uniform moment estimates and convergence in probability. This allows us to obtainusing the Yamada-Watanabe theorem - that there exists a unique strong solution. The Feller property of the semigroup as well as existence of invariant measures are then shown in Sect. 4.

The results of this work were published as part of the author's $\mathrm{PhD}$ thesis [41]. More detailed discussions of the literature as well as calculations can be found there.

\section{Preliminaries}

In this section, we provide the basic tools needed later. After establishing our notation and the assumptions the coefficients, we prove some elementary estimates for the operators associated to the coefficients. We then provide a tightness criterion for later use.

\subsection{Notation and assumptions}

For a domain $G \subset \mathbb{R}^{3}$, we use the following notational hierarchy for $L^{p}$ and Sobolev spaces:

(1) The spaces $L^{p}(G, \mathbb{R})$ of real-valued integrable (equivalence classes of) functionse.g. the components $v^{i}, B^{i}$-will be denoted as $L^{p}(G)$ or $L^{p}$ if no confusion can arise.

(2) We may sometimes use the notation $\boldsymbol{L}^{p}(G):=L^{p}\left(G ; \mathbb{R}^{3}\right)$ to denote 3-D vectorvalued integrable quantities, especially the velocity vector field and magnetic vector field $\boldsymbol{v}$ and $\boldsymbol{B}$.

(3) The divergence-free and $p$-integrable vector fields will be denoted by $\backslash$ mathbb symbols, so $\mathbb{L}^{p}(G):=\boldsymbol{L}^{p}(G) \cap \operatorname{div}^{-1}\{0\}$. Its elements $\boldsymbol{v}, \boldsymbol{B}$ satisfy $\operatorname{div} \boldsymbol{v}=\nabla \cdot \boldsymbol{v}=$ $0, \operatorname{div} \boldsymbol{B}=0$.

(4) Finally, we denote the space of the combined velocity and magnetic vector fields by $\backslash$ mathcal symbols, i.e., $\mathcal{L}^{p}(G):=\mathbb{L}^{p}(G) \times \mathbb{L}^{p}(G)$. Its elements are of the form $y=(\boldsymbol{v}, \boldsymbol{B})$.

For Sobolev spaces, we use the same notational conventions, so for example $\mathbb{H}^{k}(G):=$ $\boldsymbol{H}^{k}(G) \cap \operatorname{div}^{-1}\{0\}:=W^{k, 2}\left(G ; \mathbb{R}^{3}\right) \cap \operatorname{div}^{-1}\{0\}$ etc. Finally, if the domain of the functions is not in $\mathbb{R}^{3}$, in particular if it is a real interval (for the time variable), then we use the unchanged $L^{p}$ notation. By $\ell^{2}$, we denote the space of square-summable sequences.

For brevity, we use the following terminology when discussing the terms on the right-hand side of the tamed MHD equations: the terms involving the Laplace operator are called the linear terms, the terms involving the taming function $g_{N}$ are called taming terms and the other terms are called the nonlinear terms. Furthermore, we refer to the initial data $y_{0}=\left(\boldsymbol{v}_{0}, \boldsymbol{B}_{0}\right)$ and the force $f=\left(\boldsymbol{f}_{v}, \boldsymbol{f}_{B}\right)$ collectively as the data of the problem.

For definiteness, we want to state the following very elementary relationship between the classical homogeneous second-order Sobolev norm and the norm we use in this work (which is defined via Bessel potentials). 
Lemma 2.1 Let $\mathbb{D} \in\left\{\mathbb{R}^{3}, \mathbb{T}^{3}\right\}$ and $y \in \mathcal{H}^{2}(\mathbb{D})$. Then the following estimate holds:

$$
\|y\|_{\dot{\mathcal{V}}^{2,2}(\mathbb{D})}^{2}:=\sum_{i, j=1}^{d}\left\|\partial_{x^{i}} \partial_{x^{j}} y\right\|_{\mathcal{L}^{2}(\mathbb{D})}^{2} \leq 3^{2}\|y\|_{\mathcal{H}^{2}(\mathbb{D})}^{2} .
$$

We will use the following estimate below.

Lemma 2.2 For a function $y=(\boldsymbol{v}, \boldsymbol{B})$ such that $\left|\boldsymbol{v}\left\|\nabla \boldsymbol{v}\left|\in L^{2},\right| \boldsymbol{B}\right\| \nabla \boldsymbol{B}\right| \in L^{2}$, it holds that $y \in \mathcal{L}^{12}$ and

$$
\|y\|_{\mathcal{L}^{12}}^{4} \leq C\left(\left\|\left|\boldsymbol{v}\left\|\nabla \boldsymbol{v}\left|\left\|_{L^{2}}^{2}+\right\|\right| \boldsymbol{B}\right\| \nabla \boldsymbol{B}\right|\right\|_{L^{2}}^{2}\right)
$$

Proof By the Gagliardo-Nirenberg-Sobolev inequality for scalar functions $f \in$ $H^{1}\left(\mathbb{R}^{3}\right)$

$$
\|f\|_{L^{6}} \leq C\|\nabla f\|_{L^{2}}
$$

it follows that

$$
\begin{aligned}
\|y\|_{\mathcal{L}^{12}}^{4}=\left\||y|^{2}\right\|_{\mathcal{L}^{6}}^{2} & \leq C\left\|\nabla|y|^{2}\right\|_{L^{2}}^{2}=4 C \int \sum_{i=1}^{3}\left|\sum_{j=1}^{3}\left(v_{j} \partial_{i} v_{j}+B_{j} \partial_{i} B_{j}\right)\right|^{2} \mathrm{~d} x \\
& \leq 24 C \int \sum_{j=1}^{3}\left(\left|v_{j}\right|^{2} \sum_{i=1}^{3}\left|\partial_{i} v_{j}\right|^{2}+\left|B_{j}\right|^{2} \sum_{i=1}^{3}\left|\partial_{i} B_{j}\right|^{2}\right) \mathrm{d} x \\
& \leq 24 C\left(\left\|\left|\boldsymbol{v}\left\|\nabla \boldsymbol{v}\left|\left\|_{L^{2}}^{2}+\right\|\right| \boldsymbol{B}\right\| \nabla \boldsymbol{B}\right|\right\|_{L^{2}}^{2}\right),
\end{aligned}
$$

which proves the claim.

We make the following assumptions on our coefficients:

(H1) For any $T>0$, the function $f=\left(\boldsymbol{f}_{v}, \boldsymbol{f}_{B}\right)$ with $\boldsymbol{f}_{v}, \boldsymbol{f}_{B}:[0, T] \times \mathbb{D} \times$ $\mathbb{R}^{6} \rightarrow \mathbb{R}^{3}$ satisfies: there is a constant $C_{T, f}>0$ and a function $F_{f}(t, x) \in$ $L^{\infty}\left([0, T] ; L^{1}(\mathbb{D})\right)$

$$
\begin{aligned}
\left|\partial_{x^{j}} f(t, x, y)\right|^{2}+|f(t, x, y)|^{2} & \leq C_{T, f}|y|^{2}+F_{f}(t, x), \quad j=1,2,3, x \in \mathbb{D}, y \in \mathbb{R}^{6}, \\
\left|\partial_{y^{l}} f(t, x, y)\right| & \leq C_{T, f}, \quad l=1, \ldots, 6, x \in \mathbb{D}, y \in \mathbb{R}^{6} .
\end{aligned}
$$

(H2) For any $T>0$, for the function $\Sigma=(\sigma, \bar{\sigma})$ with $\sigma, \bar{\sigma}:[0, T] \times \mathbb{D} \rightarrow \ell^{2}\left(\mathbb{R}^{3}\right)$, there are constants $C_{\sigma, T}, C_{\bar{\sigma}, T}>0$ such that for $j=1,2,3$

$$
\sup _{t \in[0, T], x \in \mathbb{D}}\left\|\partial_{x^{j}} \sigma(t, x)\right\|_{\ell^{2}} \leq C_{\sigma, T}, \quad \sup _{t \in[0, T], x \in \mathbb{D}}\left\|\partial_{x^{j}} \overline{\boldsymbol{\sigma}}(t, x)\right\|_{\ell^{2}} \leq C_{\bar{\sigma}, T}
$$


as well as

$$
\sup _{t \in[0, T], x \in \mathbb{D}}\|\Sigma(t, x)\|_{\ell^{2}}^{2} \leq \frac{1}{36} .
$$

(H3) For any $T>0$, for the function $H=(\boldsymbol{h}, \overline{\boldsymbol{h}})$ with $\boldsymbol{h}, \overline{\boldsymbol{h}}:[0, T] \times \mathbb{D} \times \mathbb{R}^{6} \rightarrow$ $\ell^{2}\left(\mathbb{R}^{3}\right)$, there exists a constant $C_{T, H}>0$ and $F_{H}(t, x) \in L^{\infty}\left([0, T] ; L^{1}(\mathbb{D})\right)$ such that for any $0 \leq t \leq T, x \in \mathbb{D}, y \in \mathbb{R}^{6}$ and $j=1,2,3, l=1,2, \ldots, 6$

$$
\begin{aligned}
\left\|\partial_{x^{j}} H(t, x, y)\right\|_{\ell^{2}}^{2}+\|H(t, x, y)\|_{\ell^{2}}^{2} & \leq C_{T, H}|y|^{2}+F_{H}(t, x), \\
\left\|\partial_{y^{l}} H(t, x, y)\right\|_{\ell^{2}} & \leq C_{T, H} .
\end{aligned}
$$

Remark 2.3 The origin of the constant $36=4 \cdot 3^{2}$ in Assumption (H2) lies in the fact that in the places where we need the numerical value, i.e. in the proof of (18) of Lemma 2.6 as well as in the proof of Lemma 4.1, we estimate the homogeneous second-order Sobolev norm against the Bessel potential norm via Lemma 2.1 below, giving an additional factor of $9=3^{2}$. We do not claim that this value for $\Sigma$ is sharp, but we wanted to give an explicit bound that suffices to make all the calculations work.

The integrability conditions $F_{f}, F_{H} \in L^{\infty}\left([0, T] ; L^{1}(\mathbb{D})\right)$ are used in proving continuity in time, as we want to estimate

$$
\int_{s}^{t}\left\|F_{f, H}(r)\right\|_{L^{1}(\mathbb{D})} \mathrm{d} r \leq C|t-s|
$$

in the proof of Lemma 3.11 .

One could also model the equations in a way that the terms $\boldsymbol{f}_{v}$ and $\boldsymbol{h}$ in the equations of $\boldsymbol{v}$ depend only on $\boldsymbol{v}$ instead of $y$. However, this case is included in our assumptions, which are more symmetric this way.

We define the set of solenoidal test functions as

$$
\mathcal{V}:=\left\{y=(\boldsymbol{v}, \boldsymbol{B}) \mid \boldsymbol{v}, \boldsymbol{B} \in C_{c}^{\infty}\left(\mathbb{D} ; \mathbb{R}^{3}\right), \operatorname{div}(\boldsymbol{v})=\operatorname{div}(\boldsymbol{B})=0\right\}
$$

As in [40, Lemma 2.1], $\mathcal{V}$ is dense in $\mathcal{H}^{k}$ for any $k \in \mathbb{N}$. Let $P: L^{2}\left(\mathbb{D} ; \mathbb{R}^{3}\right) \rightarrow \mathbb{H}^{0}$ be the Leray-Helmholtz projection. In the case of $\mathbb{D} \in\left\{\mathbb{T}, \mathbb{R}^{3}\right\}, P$ commutes with derivative operators (cf. [35, Lemma 2.9, p. 52]) and can be restricted to a bounded linear operator

$$
\left.P\right|_{H^{m}}: H^{m} \rightarrow \mathbb{H}^{m}
$$

Furthermore, consider the tensorised projection

$$
\mathcal{P}:=P \otimes P, \quad \mathcal{P} y:=(P \otimes P)\left(\begin{array}{l}
\boldsymbol{v} \\
\boldsymbol{B}
\end{array}\right)=\left(\begin{array}{l}
P \boldsymbol{v} \\
P \boldsymbol{B}
\end{array}\right)
$$


Then $\mathcal{P}: \mathcal{L}^{2} \rightarrow \mathcal{H}^{0}$ is a bounded linear operator:

$$
\|\mathcal{P} y\|_{\mathcal{H}^{0}}^{2}=\|P \boldsymbol{v}\|_{\mathbb{H}^{0}}^{2}+\|P \boldsymbol{B}\|_{\mathbb{H}^{0}}^{2} \leq\|P\|_{L^{2} \rightarrow \mathbb{H}^{0}}^{2}\left(\|\boldsymbol{v}\|_{L^{2}}^{2}+\|B\|_{L^{2}}^{2}\right)=\|P\|_{L^{2} \rightarrow \mathbb{H}^{0}}^{2}\|y\|_{\mathcal{L}^{2}}^{2}
$$

We now define operators

$$
\begin{gathered}
\mathcal{A}(y):=\mathcal{P} \Delta y-\mathcal{P}\left(\begin{array}{l}
(\boldsymbol{v} \cdot \nabla) \boldsymbol{v}-(\boldsymbol{B} \cdot \nabla) \boldsymbol{B} \\
(\boldsymbol{v} \cdot \nabla) \boldsymbol{B}-(\boldsymbol{B} \cdot \nabla) \boldsymbol{v}
\end{array}\right)-\mathcal{P}\left(g_{N}\left(|y|^{2}\right) y\right), \\
\langle\mathcal{A}(y), \tilde{y}\rangle_{\mathcal{H}^{1}}=\langle\mathcal{A}(y),(I-\Delta) \tilde{y}\rangle_{\mathcal{H}^{0}}=\mathcal{A}_{1}(y, \tilde{y})+\mathcal{A}_{2}(y, \tilde{y})+\mathcal{A}_{3}(y, \tilde{y}),
\end{gathered}
$$

where

$$
\begin{aligned}
& \mathcal{A}_{1}(y, \tilde{y}):=\langle\mathcal{P} \Delta y,(I-\Delta) \tilde{y}\rangle_{\mathcal{H}^{0}}, \\
& \mathcal{A}_{2}(y, \tilde{y}):=-\left\langle\mathcal{P}\left(\begin{array}{l}
(\boldsymbol{v} \cdot \nabla) \boldsymbol{v}-(\boldsymbol{B} \cdot \nabla) \boldsymbol{B} \\
(\boldsymbol{v} \cdot \nabla) \boldsymbol{B}-(\boldsymbol{B} \cdot \nabla) \boldsymbol{v}
\end{array}\right),(I-\Delta) \tilde{y}\right\rangle_{\mathcal{H}^{0}}, \\
& \mathcal{A}_{3}(y, \tilde{y}):=-\left\langle\mathcal{P} g_{N}\left(|y|^{2}\right) y,(I-\Delta) \tilde{y}\right\rangle_{\mathcal{H}^{0}} .
\end{aligned}
$$

For the noise terms, we define $\Sigma(t, x):=\left(\begin{array}{c}\boldsymbol{\sigma}_{k}(t, x) \\ \overline{\boldsymbol{\sigma}}_{k}(t, x)\end{array}\right)_{k \in \mathbb{N}} \in \ell^{2}\left(\mathbb{R}^{6}\right)$ and for $y=\left(\begin{array}{c}\boldsymbol{v} \\ \boldsymbol{B}\end{array}\right)$

$$
\left(\Sigma_{k}(t, x) \cdot \nabla\right) y:=\left(\begin{array}{c}
\left(\boldsymbol{\sigma}_{k}(t, x) \cdot \nabla\right) \boldsymbol{v} \\
\left(\overline{\boldsymbol{\sigma}}_{k}(t, x) \cdot \nabla\right) \boldsymbol{B}
\end{array}\right) .
$$

Similarly, we define $H_{k}(t, y):=\left(\begin{array}{l}\boldsymbol{h}_{k}(t, y) \\ \overline{\boldsymbol{h}}_{k}(t, y)\end{array}\right)$, and

$$
\mathcal{B}_{k}(t, x, y):=\mathcal{P}\left(\left(\Sigma_{k}(t, x) \cdot \nabla\right) y\right)+\mathcal{P} H_{k}(t, x, y) .
$$

Finally, let $\left\{W_{t}^{k} \mid t \in \mathbb{R}_{+}, k \in \mathbb{N}\right\},\left\{\bar{W}_{t}^{k} \mid t \in \mathbb{R}_{+}, k \in \mathbb{N}\right\}$ be two independent sequences of independent Brownian motions, and define

$$
\mathcal{W}_{t}^{k}:=\left(\begin{array}{c}
W_{t}^{k} \\
\bar{W}_{t}^{k}
\end{array}\right)
$$

The stochastic integral in our equations is defined by

$$
\int_{0}^{t} \mathcal{B}_{k}(s, x, y) \mathrm{d} \mathcal{W}_{s}^{k}:=\left(\begin{array}{l}
\int_{0}^{t} P\left(\boldsymbol{\sigma}_{k}(s, x) \cdot \nabla\right) \boldsymbol{v}+P \boldsymbol{h}_{k}(s, x, y) \mathrm{d} W_{s}^{k} \\
\int_{0}^{t} P\left(\overline{\boldsymbol{\sigma}}_{k}(s, x) \cdot \nabla\right) \boldsymbol{B}+P \overline{\boldsymbol{h}}_{k}(s, x, y) \mathrm{d} \bar{W}_{s}^{k}
\end{array}\right) .
$$

The Brownian motions $W$ and $\bar{W}$ can be understood as independent cylindrical Brownian motions on the space $\ell^{2}$. Similarly, $\mathcal{W}$ is a cylindrical Brownian motion on the 
space $\ell^{2} \times \ell^{2}$. For $y \in \mathcal{H}^{m}, m=1,2, \mathcal{B}(t, x, y(x))$ can be understood as a linear operator

$$
\mathcal{B}(t, \cdot, y): \ell^{2} \times \ell^{2} \rightarrow \mathcal{H}^{m-1}
$$

To make this clear, we note that if we take the canonical basis of $\ell^{2}$, i.e. orthonormal basis consisting of the sequences $e_{1}:=(1,0,0, \ldots), e_{2}:=(0,1,0,0, \ldots), \ldots$, then the system

$$
\left\{\left(\begin{array}{c}
e_{k} \\
0
\end{array}\right) \in \ell^{2} \times \ell^{2} \mid k \in \mathbb{N}\right\} \cup\left\{\left(\begin{array}{c}
0 \\
e_{k}
\end{array}\right) \in \ell^{2} \times \ell^{2} \mid k \in \mathbb{N}\right\}
$$

forms an orthonormal basis of $\ell^{2} \times \ell^{2}$. Then we define

$$
\mathcal{B}(t, \cdot, y)\left(\begin{array}{c}
e_{k} \\
0
\end{array}\right)(x):=\left(\begin{array}{c}
P\left(\sigma_{k}(t, x) \cdot \nabla\right) \boldsymbol{v}(x)+P \boldsymbol{h}_{k}(t, x, y(x)) \\
0
\end{array}\right) \in \mathcal{H}^{m-1},
$$

and

$$
\mathcal{B}(t, \cdot, y)\left(\begin{array}{c}
0 \\
e_{k}
\end{array}\right)(x):=\left(\begin{array}{c}
0 \\
P\left(\overline{\boldsymbol{\sigma}}_{k}(t, x) \cdot \nabla\right) \boldsymbol{v}(x)+P \overline{\boldsymbol{h}}_{k}(t, x, y(x))
\end{array}\right) \in \mathcal{H}^{m-1} .
$$

It turns out that $\mathcal{B}$ is even a Hilbert-Schmidt operator, i.e. $\mathcal{B}(t, \cdot, y) \in L_{2}\left(\ell^{2} \times\right.$ $\ell^{2} ; \mathcal{H}^{m-1}$ ), as will be proved below in Lemma 2.6. Hence we are in the usual framework of stochastic analysis on Hilbert spaces, cf. [10] or [26].

We can now formulate Eq. (1) in the following abstract form as an evolution equation:

$$
\left\{\begin{array}{l}
\mathrm{d} y(t)=[\mathcal{A}(y(t))+\mathcal{P} f(t, y(t))] \mathrm{d} t+\sum_{k=1}^{\infty} \mathcal{B}_{k}(t, y(t)) \mathrm{d} \mathcal{W}_{t}^{k}, \\
y(0)=y_{0} \in \mathcal{H}^{1}
\end{array}\right.
$$

\subsection{Estimates on the operators $\mathcal{A}$ and $\mathcal{B}$}

In this section, we will collect important but elementary estimates on the operators $\mathcal{A}$ and $\mathcal{B}$. These play an important role in deriving the a priori estimates for the STMHD equations. The first lemma is concerned with estimates for the case of testing with the solution $y$ itself. 
Lemma 2.4 (Estimates for $\mathcal{A}$ ) Let $y \in \mathcal{H}^{2}$. Then the following estimates hold true

$$
\begin{aligned}
\|\mathcal{A}(y)\|_{\mathcal{H}^{0}} \leq & C\left(1+\|y\|_{\mathcal{H}^{0}}^{6}+\|y\|_{\mathcal{H}^{2}}^{2}\right) \\
\langle\mathcal{A}(y), y\rangle_{\mathcal{H}^{0}}= & -\|\nabla y\|_{\mathcal{H}^{0}}^{2}-\left\|\sqrt{g_{N}\left(|y|^{2}\right)}|y|\right\|_{\mathcal{L}^{2}}^{2} \\
\leq & -\|\nabla y\|_{\mathcal{H}^{0}}^{2}-\|y\|_{\mathcal{L}^{4}}^{2}+C N\|y\|_{\mathcal{H}^{0}}^{2}, \\
\langle\mathcal{A}(y), y\rangle_{\mathcal{H}^{1}} \leq & -\frac{1}{2}\|y\|_{\mathcal{H}^{2}}^{2}-\left(\||\boldsymbol{v}| \cdot|\nabla \boldsymbol{v}|\|_{L^{2}}^{2}+\||\boldsymbol{B}| \cdot|\nabla \boldsymbol{B}|\|_{L^{2}}^{2}\right. \\
& \left.+\||\boldsymbol{v}| \cdot|\nabla \boldsymbol{B}|\|_{L^{2}}^{2}+\||\boldsymbol{B}| \cdot|\nabla \boldsymbol{v}|\|_{L^{2}}^{2}\right) \\
& +(2 N+1)\|\nabla y\|_{\mathcal{H}^{0}}^{2}+\|y\|_{\mathcal{H}^{0}}^{2} .
\end{aligned}
$$

Proof This follows in the same way as [42, Lemma 2.2, p. 9].

The next lemma deals with the case of testing the right-hand side of our equation with another, arbitrary, compactly supported test function.

Lemma 2.5 (Estimates for $\mathcal{A}$ and $\mathcal{B})$ Let $\tilde{y} \in \mathcal{V}$ with compact support $\operatorname{supp}(\tilde{y}) \subset$ $\mathcal{O}:=\{x \in \mathbb{D}|| x \mid \leq m\}$ for some $m \in \mathbb{N}$. Let $T>0$. Then for any $y, y^{\prime} \in \mathcal{H}^{2}$ and $t \in[0, T]$, we have

$$
\begin{aligned}
\left|\langle\mathcal{A}(y), \tilde{y}\rangle_{\mathcal{H}^{1}}\right| & \leq C_{\tilde{y}}\left(1+\|y\|_{\mathcal{L}^{3}(\mathcal{O})}^{3}\right), \\
\left\|\langle\mathcal{B} .(t, y), \tilde{y}\rangle_{\mathcal{H}^{1}}\right\|_{\ell^{2}}^{2} & \leq C_{\tilde{y}, \Sigma, H, T}\left(\left\|F_{H}(t)\right\|_{L^{1}(\mathbb{D})}+\|y\|_{\mathcal{L}^{2}(\mathcal{O})}^{2}\right),
\end{aligned}
$$

and

$$
\left|\left\langle\mathcal{A}(y)-\mathcal{A}\left(y^{\prime}\right), \tilde{y}\right\rangle_{\mathcal{H}^{1}}\right| \leq C_{\tilde{y}, N}\left\|y-y^{\prime}\right\|_{\mathcal{L}^{2}}\left(1+\|y\|_{\mathcal{H}^{1}}^{2}+\left\|y^{\prime}\right\|_{\mathcal{H}^{1}}^{2}\right) .
$$

Proof The first inequality follows easily from the following calculations:

$$
\mathcal{A}_{1}(y, \tilde{y})=\langle y,(I-\Delta) \Delta \tilde{y}\rangle_{\mathcal{H}^{0}} \leq\|y\|_{\mathcal{L}^{2}(\mathcal{O})}\|(I-\Delta) \Delta \tilde{y}\|_{\mathcal{H}^{0}} \leq C_{\tilde{y}}\|y\|_{L^{3}(\mathcal{O})}\|\tilde{y}\|_{\mathcal{H}^{4}},
$$

where the constant $C_{\tilde{y}}$ depends on the domain $\mathcal{O}$, and hence on $\tilde{y}$. For the next term we find

$$
\begin{aligned}
\mathcal{A}_{2}(y, \tilde{y}) \leq & \left(\left(\|\boldsymbol{v}\|_{L^{2}(\mathcal{O})}^{2}+\|\boldsymbol{B}\|_{L^{2}(\mathcal{O})}^{2}\right) \cdot \sup _{x \in \mathbb{D}}|\nabla(I-\Delta) \tilde{\boldsymbol{v}}(x)|\right. \\
& \left.+2\|\boldsymbol{v}\|_{L^{2}(\mathcal{O})}\|\boldsymbol{B}\|_{L^{2}(\mathcal{O})} \cdot \sup _{x \in \mathbb{D}}|\nabla(I-\Delta) \tilde{\boldsymbol{B}}(x)|\right) \\
\leq & 2\|y\|_{\mathcal{L}^{2}(\mathcal{O})}^{2} \cdot \sup _{x \in \mathbb{D}}|\nabla(I-\Delta) \tilde{\boldsymbol{y}}(x)| \\
\leq & C_{\tilde{y}}\|y\|_{\mathcal{L}^{3}(\mathcal{O})}^{2} \cdot \sup _{x \in \mathbb{D}}|\nabla(I-\Delta) \tilde{\boldsymbol{y}}(x)| .
\end{aligned}
$$


Finally,

$$
\mathcal{A}_{3}(y, \tilde{y}) \leq\left\|g_{N}\left(|y|^{2}\right) y\right\|_{\mathcal{L}^{1}(\mathcal{O})} \cdot \sup _{x \in \mathbb{D}}|(I-\Delta) \tilde{y}(x)| \leq C\|y\|_{\mathcal{L}^{3}(\mathcal{O})}^{3} \sup _{x \in \mathbb{D}}|(I-\Delta) \tilde{y}(x)| .
$$

Combining the above estimates yields (14).

For the second estimate (15), we have by the boundedness of the Leray-Helmholtz projections

$$
\begin{aligned}
& \left\|\langle\mathcal{B} .(t, y), \tilde{y}\rangle_{\mathcal{H}^{1}}\right\|_{\ell^{2}}^{2} \leq\left\|\langle(\Sigma \cdot \nabla) y,(I-\Delta) \tilde{y}\rangle_{\mathcal{L}^{2}}+\langle H(t, y),(I-\Delta) \tilde{y}\rangle_{\mathcal{L}^{2}}\right\|_{\ell^{2}}^{2} \\
& \quad \leq 2\left(\sum_{k}\left|\left\langle\left(\Sigma_{k} \cdot \nabla\right) y,(I-\Delta) \tilde{y}\right\rangle_{\mathcal{L}^{2}}\right|^{2}+\left|\left\langle H_{k}(t, y),(I-\Delta) \tilde{y}\right\rangle_{\mathcal{L}^{2}}\right|^{2}\right) .
\end{aligned}
$$

The first term can be estimated using the definitions of the scalar products and norms involved, integration by parts, the product rule and Jensen's inequality ( $\lambda$ denotes the Lebesgue measure):

$$
\begin{aligned}
& \sum_{k}\left|\left\langle\left(\Sigma_{k} \cdot \nabla\right) y,(I-\Delta) \tilde{y}\right\rangle_{\mathcal{L}^{2}}\right|^{2} \\
& =\sum_{k} \mid \int_{\mathcal{O}} \sum_{j, l=1}^{3} v^{l}\left(\partial_{x^{j}} \sigma_{k}^{j}\right)(I-\Delta) \tilde{v}^{l}+v^{l} \sigma_{k}^{j}(I-\Delta) \partial_{x^{j}} \tilde{v}^{l} \\
& \quad+B^{l}\left(\partial_{x^{j}} \bar{\sigma}_{k}^{j}\right)(I-\Delta) \tilde{B}^{l}+\left.B^{l} \bar{\sigma}_{k}^{j}(I-\Delta) \partial_{x^{j}} \tilde{B}^{l} \mathrm{~d} x\right|^{2} \\
& \leq 36 \lambda(\mathcal{O}) \sum_{k} \int_{\mathcal{O}} \sum_{j, l=1}^{3}\left|v^{l}\left(\partial_{x^{j}} \sigma_{k}^{j}\right)(I-\Delta) \tilde{v}^{l}\right|^{2}+\left|v^{l} \sigma_{k}^{j}(I-\Delta) \partial_{x^{j}} \tilde{v}^{l}\right|^{2} \\
& \quad+\left|B^{l}\left(\partial_{x^{j}} \bar{\sigma}_{k}^{j}\right)(I-\Delta) \tilde{B}^{l}\right|^{2}+\left|B^{l} \bar{\sigma}_{k}^{j}(I-\Delta) \partial_{x^{j}} \tilde{B}^{l}\right|^{2} \mathrm{~d} x \\
& \leq 36 \lambda(\mathcal{O})\left(\sup _{t \in[0, T], x \in \mathbb{D}}\left\|\nabla_{x} \Sigma .(t, x)\right\|_{\ell^{2}}^{2} \sup _{x \in \mathbb{D}}|(I-\Delta) \tilde{y}(x)|^{2}\right. \\
& \left.\quad+\sup _{t \in[0, T], x \in \mathbb{D}}\|\Sigma .(t, x)\|_{\ell^{2}}^{2} \sup _{x \in \mathbb{D}}|\nabla(I-\Delta) \tilde{y}(x)|^{2}\right)\|y\|_{\mathcal{L}^{2}(\mathcal{O})}^{2}
\end{aligned}
$$

We estimate this further by using Assumption (H2), arriving at

$$
\sum_{k}\left|\left\langle\left(\Sigma_{k} \cdot \nabla\right) y,(I-\Delta) \tilde{y}\right\rangle_{\mathcal{L}^{2}}\right|^{2} \leq C_{\Sigma, T, \tilde{y}}\|y\|_{\mathcal{L}^{2}(\mathcal{O})}^{2} .
$$


Similarly, for the second term we find

$$
\begin{aligned}
\sum_{k} & \left|\left\langle H_{k}(t, y),(I-\Delta) \tilde{y}\right\rangle_{\mathcal{L}^{2}}\right|^{2} \\
\leq & 2 \lambda(O) \sum_{k} \int_{\mathcal{O}}\left|\left\langle\boldsymbol{h}_{k}(t, x, y),(I-\Delta) \tilde{\boldsymbol{v}}(x)\right\rangle_{\mathbb{R}^{3}}\right|^{2} \\
& +\left|\left\langle\overline{\boldsymbol{h}}_{k}(t, x, y),(I-\Delta) \tilde{\boldsymbol{B}}(x)\right\rangle_{\mathbb{R}^{3}}\right|^{2} \mathrm{~d} x \\
\leq & 2 \lambda(O)\left(\left.\sup _{x \in \mathbb{D}}|(I-\Delta) \tilde{\boldsymbol{v}}(x)|^{2} \int_{\mathcal{O}}\|\boldsymbol{h} \cdot(t, x, y)\|\right|_{\ell^{2}} ^{2} \mathrm{~d} x\right. \\
& \left.+\sup _{x \in \mathbb{D}}|(I-\Delta) \tilde{\boldsymbol{B}}(x)|^{2} \int_{\mathcal{O}}\|\overline{\boldsymbol{h}} \cdot(t, x, y)\|_{\ell^{2}}^{2} \mathrm{~d} x\right) \\
\leq & 2 \lambda(O) C_{T, H} \sup _{x \in \mathbb{D}}|(I-\Delta) \tilde{y}(x)|^{2} \int_{\mathcal{O}}|y(x)|^{2}+F_{H}(t, x) \mathrm{d} x \\
\leq & C_{H, T, \tilde{y}}\left(\|y\|_{\mathcal{L}^{2}(\mathcal{O})}^{2}+\left\|F_{H}(t)\right\|_{L^{1}(\mathbb{D})}\right) .
\end{aligned}
$$

Thus, altogether we have proved (15).

Estimate (16) follows in the same way as [39, Lemma 2.4, Equation (2.13), pp. 218 f.].

Lemma 2.6 (Estimates for $\mathcal{B}$ ) For any $T>0,0 \leq t \leq T$ and $y \in \mathcal{H}^{2}$, the following estimates hold:

$$
\begin{aligned}
\|\mathcal{B}(t, y)\|_{L_{2}\left(\ell^{2} \times \ell^{2} ; \mathcal{H}^{0}\right)}^{2} & \leq \frac{1}{2}\|y\|_{\mathcal{H}^{1}}^{2}+C_{T, H}\|y\|_{\mathcal{H}^{0}}^{2}+\left\|F_{H}(t)\right\|_{L^{1}(\mathbb{D})}, \\
\|\mathcal{B}(t, y)\|_{L_{2}\left(\ell^{2} \times \ell^{2} ; \mathcal{H}^{1}\right)}^{2} & \leq \frac{1}{2}\|y\|_{\mathcal{H}^{2}}^{2}+C_{T, H, \Sigma}\|y\|_{\mathcal{H}^{1}}^{2}+C\left\|F_{H}(t)\right\|_{L^{1}(\mathbb{D})} .
\end{aligned}
$$

Proof To calculate the Hilbert-Schmidt norm (cf. [26, Definition B.0.5, p. 217]), we take the orthonormal basis of $\ell^{2} \times \ell^{2}$ from Eq. (8) and enumerate it as a set $\left\{\hat{e}_{l}\right\}_{l \in \mathbb{N}}$. Then by definition, for $m=0,1$,

$$
\begin{aligned}
& \|\mathcal{B}(t, y)\|_{L_{2}\left(\ell^{2} \times \ell^{2} ; \mathcal{H}^{m}\right)}^{2} \\
& =\sum_{l \in \mathbb{N}}\left\|\mathcal{B}(t, y) \hat{e}_{l}\right\|_{\mathcal{H}^{m}}^{2}=\sum_{k \in \mathbb{N}}\left\|\mathcal{B}(t, y)\left(\begin{array}{c}
e_{k} \\
0
\end{array}\right)\right\|_{\mathcal{H}^{m}}^{2}+\sum_{k \in \mathbb{N}}\left\|\mathcal{B}(t, y)\left(\begin{array}{c}
0 \\
e_{k}
\end{array}\right)\right\|_{\mathcal{H}^{m}}^{2} \\
& =\sum_{k \in \mathbb{N}}\left\|\mathcal{B}_{k}(t, y)\right\|_{\mathcal{H}^{m}}^{2} .
\end{aligned}
$$


The first inequality follows from Assumptions (H2) and (H3):

$$
\begin{aligned}
& \|\mathcal{B}(t, y)\|_{L_{2}\left(\ell^{2} \times \ell^{2} ; \mathcal{H}^{0}\right)}^{2}=\sum_{k} \int_{\mathbb{D}}\left|\mathcal{B}_{k}(t, x, y)\right|^{2} \mathrm{~d} x \\
& \quad \leq 2 \int_{\mathbb{D}}\|\Sigma(t, x)\|_{\ell^{2}}^{2}|\nabla y|^{2}+\left\|H_{k}(t, x, y)\right\|_{\ell^{2}}^{2} \mathrm{~d} x \\
& \quad \leq 2\left(\sup _{t \in[0, T], x \in \mathbb{D}}\|\Sigma(t, x)\|_{\ell^{2}}^{2}\right) \int_{\mathbb{D}}|\nabla y|^{2} \mathrm{~d} x+2 C_{T, H} \int_{\mathbb{D}}|y|^{2}+F_{H}(t, x) \mathrm{d} x \\
& \quad \leq \frac{1}{2}\|y\|_{\mathcal{H}^{1}}^{2}+\left(2 C_{T, H}-\frac{1}{2}\right)\|y\|_{\mathcal{H}^{0}}^{2}+\left\|F_{H}(t)\right\|_{L^{1}(\mathbb{D})} .
\end{aligned}
$$

For the second inequality, we note that, using the commutativity of derivatives and $\mathcal{P}$ as well as the chain rule, we find

$$
\begin{aligned}
\partial_{x^{j}} \mathcal{B}_{k}(t, x, y)= & \partial_{x^{j}} \mathcal{P}\left[\left(\Sigma_{k}(t, x) \cdot \nabla\right) y+H_{k}(t, x, y)\right] \\
= & \mathcal{P} \partial_{x^{j}}\left[\left(\Sigma_{k}(t, x) \cdot \nabla\right) y+H_{k}(t, x, y)\right] \\
= & \mathcal{P}\left[\left(\partial_{x^{j}} \Sigma_{k}(t, x) \cdot \nabla\right) y+\left(\Sigma_{k}(t, x) \cdot \nabla\right) \partial_{x^{j}} y+\left(\partial_{x^{j}} H_{k}\right)(t, x, y)\right. \\
& \left.+\sum_{i=1}^{6} \partial_{y^{i}} H_{k}(t, x, y) \partial_{x^{j}} y^{i}\right] .
\end{aligned}
$$

Therefore, employing Assumptions (H2), (H3), as well as Eq. (5), we find

$$
\begin{aligned}
\|\nabla \mathcal{B}(t, y)\|_{L_{2}\left(\ell^{2} \times \ell^{2} ; \mathcal{H}^{0}\right)}^{2} & \leq \sum_{k} \sum_{j=1}^{3} \int_{\mathbb{D}} 2\left|\left(\Sigma_{k}(t, x) \cdot \nabla\right) \partial_{x^{j}} y\right|^{2}+2 \mid\left(\partial_{x^{j}} \Sigma_{k}(t, x) \cdot \nabla\right) y+ \\
& +\left(\partial_{x^{j}} H_{k}\right)(t, x, y)+\left.\sum_{i=1}^{6} \partial_{y^{i}} H_{k}(t, x, y) \partial_{x^{j}} y^{i}\right|^{2} \mathrm{~d} x \\
\leq & 2 \int_{\mathbb{D}} \sum_{i, j=1}^{3}\|\Sigma(t, x)\|_{\ell^{2}}^{2}\left|\partial_{x^{i}} \partial_{x^{j}} y\right|^{2} \mathrm{~d} x+6 \int_{\mathbb{D}} \sum_{j=1}^{3}\left\|\partial_{x^{j}} \Sigma(t, x)\right\|_{\ell^{2}}^{2}|\nabla y|^{2} \\
& +\left\|\left(\partial_{x^{j}} H_{k}\right)(t, x, y)\right\|_{\ell^{2}}^{2}+6 \sum_{i=1}^{6}\left\|\partial_{y^{i}} H(t, x, y)\right\|_{\ell^{2}}^{2}\left|\partial_{x^{j}} y^{i}\right|^{2} \mathrm{~d} x \\
\leq & 2 \cdot 3^{2} \sup _{t \in[0, T], x \in \mathbb{D}}\|\Sigma(t, x)\|_{\ell^{2}}^{2}\|y\|_{\mathcal{H}^{2}}^{2}+6\left(C_{\Sigma}\|\nabla y\|_{\mathcal{H}^{0}}^{2}\right. \\
& \left.+C_{T, H}\|y\|_{\mathcal{H}^{0}}^{2}+\left\|F_{H}(t)\right\|_{L^{1}(\mathbb{D})}+C_{T, H}\|\nabla y\|_{\mathcal{H}^{0}}^{2}\right) \\
\leq & \frac{1}{2}\|y\|_{\mathcal{H}^{2}}^{2}+C_{T, H, \Sigma}\left(\|\nabla y\|_{\mathcal{H}^{0}}^{2}+\|y\|_{\mathcal{H}^{0}}^{2}+\left\|F_{H}(t)\right\|_{L^{1}(\mathbb{D})}\right)
\end{aligned}
$$

which, together with (17), implies (18). 


\subsection{A tightness condition}

This section provides a tightness condition for the path space of the solutions to the stochastic tamed MHD equations. As the case of periodic boundary conditions can be treated in the same way, we will focus on the case of the full space $\mathbb{R}^{3}$.

Let $\mathbb{D}=\mathbb{R}^{3}$. We endow the space $\mathcal{H}_{\text {loc }}^{0}$ of locally $L^{2}$-integrable and divergence-free vector fields with the following Fréchet metric: for $y, z \in \mathcal{H}_{\mathrm{loc}}^{0}$

$$
\rho(y, z):=\sum_{m \in \mathbb{N}} 2^{-m}\left(\left[\int_{|x| \leq m}|y(x)-z(x)|^{2} \mathrm{~d} x\right]^{1 / 2} \wedge 1\right) .
$$

Then the space $\left(\mathcal{H}_{\text {loc }}^{0}, \rho\right)$ is a Polish space and $\mathcal{H}^{0} \subset \mathcal{H}_{\text {loc }}^{0}$.

Now set $\mathcal{X}:=C\left(\mathbb{R}_{+} ; \mathcal{H}_{\text {loc }}^{0}\right)$ and endow it with the metric

$$
\rho \mathcal{X}(y, z):=\sum_{m=1}^{\infty} 2^{-m}\left(\sup _{t \in[0, m]} \rho(y(t), z(t)) \wedge 1\right) .
$$

We fix a complete orthonormal basis $\mathcal{E}:=\left\{e_{k} \mid k \in \mathbb{N}\right\} \subset \mathcal{V}$ of $\mathcal{H}^{1}$ such that $\operatorname{span}\{\mathcal{E}\}$ is a dense subset of $\mathcal{H}^{3}$ and-in the case $\mathbb{D}=\mathbb{T}^{3}$ - such that it is also an orthogonal basis of $\mathcal{H}^{0}$. Given $y \in \mathcal{H}^{0}, z \in \mathcal{H}^{2}$, the inner product $\langle y, z\rangle_{\mathcal{H}^{1}}$ is understood in the generalised sense:

$$
\langle y, z\rangle_{\mathcal{H}^{1}}=\langle y,(I-\Delta) z\rangle_{\mathcal{H}^{0}} .
$$

This works in accordance to our choice of evolution triple

$$
\mathcal{H}^{2} \subset \mathcal{H}^{1} \subset \mathcal{H}^{0}
$$

The next lemma provides conditions for a set of $\mathcal{X}$ to be relatively compact, similar to the classical theorem of Arzelá and Ascoli. This compactness condition can then be turned into a tightness condition.

Lemma 2.7 (Compactness in $\mathcal{X}$ ) Let $K \subset \mathcal{X}$ satisfy the following conditions: for every $T>0$

(i) $\sup _{y \in K} \sup _{s \in[0, T]}\|y(s)\|_{\mathcal{H}^{1}}<\infty$,

(ii) $\lim _{\delta \rightarrow 0} \sup _{y \in K} \sup _{s, t \in[0, T],|t-s|<\delta}\left|\langle y(t)-y(s), e\rangle_{\mathcal{H}^{1}}\right|=0$ for all $e \in \mathcal{E}$.

Then $K$ is relatively compact in $\mathcal{X}$.

Proof A proof of this statement can be found in [39, Lemma 2.6, pp. $221 \mathrm{f}$.].

Lemma 2.8 (Tightness in $\mathcal{X})$ Let $\left(\mu_{n}\right)_{n \in \mathbb{N}}$ be a family of probability measures on $(\mathcal{X}, \mathcal{B}(\mathcal{X}))$ and assume that

(i) For all $T>0$

$$
\lim _{R \rightarrow \infty} \sup _{n \in \mathbb{N}} \mu_{n}\left\{y \in \mathcal{X} \mid \sup _{s \in[0, T]}\|y(s)\|_{\mathcal{H}^{1}}>R\right\}=0
$$


(ii) For all $e \in \mathcal{E}$ and any $\varepsilon, T>0$

$$
\lim _{\delta \downarrow 0} \sup _{n \in \mathbb{N}} \mu_{n}\left\{y \in \mathcal{X}\left|\sup _{s, t \in[0, T],|s-t| \leq \delta}\right|\langle y(t)-y(s), e\rangle_{\mathcal{H}^{1}} \mid>\varepsilon\right\}=0 .
$$

Then $\left(\mu_{n}\right)_{n \in \mathbb{N}}$ is tight on $(\mathcal{X}, \mathcal{B}(\mathcal{X}))$.

Proof This follows in the same way as [39, Lemma 2.7, pp. $222 \mathrm{f}$.]

\section{Existence and uniqueness of strong solutions}

In this section, we prove the main results of this paper, namely that there exists a unique strong solution to the STMHD equations. This is done by using a FaedoGalerkin approximation to get a weak solution and then proving pathwise uniqueness and employing the celebrated Yamada-Watanabe theorem to conclude existence of a unique strong solution.

\subsection{Weak and strong solutions}

For a metric space $\mathcal{U}$, we denote the set of all probability measures on $\mathcal{U}$ by $\mathcal{P}(\mathcal{U})$.

Definition 3.1 We say Eq. (9) has a weak solution with initial law $\vartheta \in \mathcal{P}\left(\mathcal{H}^{1}\right)$ if there exists a stochastic basis $\left(\Omega, \mathcal{F}, P,\left(\mathcal{F}_{t}\right)_{t \geq 0}\right)$, an $\mathcal{H}^{1}$-valued, $\left(\mathcal{F}_{t}\right)_{t}$-adapted process $y$ and two independent infinite sequences of independent standard $\left(\mathcal{F}_{t}\right)$-Brownian motions $\left\{\mathcal{W}^{k}(t)=\left(\begin{array}{l}W^{k}(t) \\ \bar{W}^{k}(t)\end{array}\right) \mid t \geq 0, k \in \mathbb{N}\right\}$ such that

(i) $y(0)$ has law $\vartheta$ in $\mathcal{H}^{1}$;

(ii) For $P$-a.e. $\omega \in \Omega$ and every $T>0, y(\cdot, \omega) \in C\left([0, T] ; \mathcal{H}^{1}\right) \cap L^{2}\left([0, T] ; \mathcal{H}^{2}\right)$;

(iii) It holds that in $\mathcal{H}^{0}$

$$
y(t)=y_{0}+\int_{0}^{t}[\mathcal{A}(y(t))+\mathcal{P} f(t, y(t))] \mathrm{d} t+\sum_{k=1}^{\infty} \int_{0}^{t} \mathcal{B}_{k}(t, y(t)) \mathrm{d} \mathcal{W}_{t}^{k},
$$

for all $t \geq 0, P$-a.s.

The solution is denoted by $\left(\Omega, \mathcal{F}, P,\left(\mathcal{F}_{t}\right)_{t \geq 0} ; \mathcal{W} ; y\right)$.

Under the Assumptions (H1)-(H3) in this section, the above integrals are all welldefined by Eq. (10).

For weak solutions, there are several notions of uniqueness. In this work, we are concerned mostly with pathwise uniqueness.

Definition 3.2 We say that pathwise uniqueness holds for Eq. (9) if, whenever we are given two weak solutions on the same stochastic basis with the same Brownian motion,

$$
\left(\Omega, \mathcal{F}, P,\left(\mathcal{F}_{t}\right)_{t \geq 0} ; \mathcal{W} ; y\right),\left(\Omega, \mathcal{F}, P,\left(\mathcal{F}_{t}\right)_{t \geq 0} ; \mathcal{W} ; y^{\prime}\right)
$$

the condition $P\left\{y(0)=y^{\prime}(0)\right\}=1$ implies $P\left\{y(t)=y^{\prime}(t), \forall t \geq 0\right\}=1$. 
The following proposition links existence of weak solutions to existence of a solution to a corresponding martingale problem.

Proposition 3.3 Let $\mathcal{E}$ and $\mathcal{X}$ be given as in Sect. 2.3. For $\vartheta \in \mathcal{P}\left(\mathcal{H}^{1}\right)$, the following are equivalent:

(i) There exists a weak solution to Eq. (9) with initial law $\vartheta$;

(ii) There exists a measure $P_{\vartheta} \in \mathcal{P}(\mathcal{X})$ with the following property: for $P_{\vartheta}$-almost all $y \in \mathcal{X}$ and any $T>0$, the real-valued process defined by

$$
y \in L^{\infty}\left([0, T] ; \mathcal{H}^{1}\right) \cap L^{2}\left([0, T] ; \mathcal{H}^{2}\right),
$$

and for any $\varphi \in C_{0}^{\infty}(\mathbb{R})$ and any $e \in \mathcal{E}$

$$
\begin{aligned}
M_{e}^{\varphi}(t, y):= & \varphi\left(\langle y(t), e\rangle_{\mathcal{H}^{1}}\right)-\varphi\left(\langle y(0), e\rangle_{\mathcal{H}^{1}}\right) \\
& -\int_{0}^{t} \varphi^{\prime}\left(\langle y(s), e\rangle_{\mathcal{H}^{1}}\right) \cdot\langle\mathcal{A}(y(s)), e\rangle_{\mathcal{H}^{1}} \mathrm{~d} s \\
& -\int_{0}^{t} \varphi^{\prime}\left(\langle y(s), e\rangle_{\mathcal{H}^{1}}\right) \cdot\langle f(s, y(s)), e\rangle_{\mathcal{H}^{1}} \mathrm{~d} s \\
& -\frac{1}{2} \int_{0}^{t} \varphi^{\prime \prime}\left(\langle y(s), e\rangle_{\mathcal{H}^{1}}\right) \cdot \|\left\langle\mathcal{B}(s, y(s)), e \|_{\ell^{2}} \mathrm{~d} s\right.
\end{aligned}
$$

is a continuous local $P_{\vartheta}$-martingale with respect to $\left(\mathcal{B}_{t}(\mathcal{X})\right)_{t}$, where $\mathcal{B}_{t}(\mathcal{X})$ denotes the sub $\sigma$-algebra of $\mathcal{X}$ up to time $t$.

Proof For a proof of this statement, cf. [39, Section 6.1, pp. $257 \mathrm{ff}$.$] .$

To be able to define the notion of strong solutions to Eq. (9), we need a canonical realisation of an infinite sequence of independent standard Brownian motions on a Polish space. To this end, consider the space $C\left(\mathbb{R}_{+}, \mathbb{R}\right)$ of continuous functions on $\mathbb{R}_{+}$with the metric

$$
\tilde{\rho}\left(w, w^{\prime}\right):=\sum_{k=1}^{\infty} 2^{-k}\left(\sup _{t \in[0, k]}\left|w(t)-w^{\prime}(t)\right| \wedge 1\right)
$$

We define the product space $\mathbb{W}:=\prod_{j=1}^{\infty} C\left(\mathbb{R}_{+} ; \mathbb{R}\right)$ and endow it with the metric

$$
\rho_{\mathbb{W}}\left(w, w^{\prime}\right):=\sum_{j=1}^{\infty} 2^{-j}\left(\tilde{\rho}\left(w^{j}, w^{\prime j}\right) \wedge 1\right), \quad w=\left(w^{1}, w^{2}, \ldots\right), \quad w^{\prime}=\left(w^{\prime 1}, w^{\prime 2}, \ldots\right) .
$$

The space $\left(\mathbb{W}, \rho_{\mathbb{W}}\right)$ is a Polish space. We denote the $\sigma$-algebra up to time $t$ by $\mathcal{B}_{t}(\mathbb{W}) \subset$ $\mathcal{B}(\mathbb{W})$ and endow $(\mathbb{W}, \mathcal{B}(\mathbb{W})$ ) with the Wiener measure $\mathbb{P}$ such that the coordinate process

$$
w(t):=\left(w^{1}(t), w^{2}(t), \ldots\right)
$$


is an infinite sequence of independent standard $\left(\mathcal{B}_{t}(\mathbb{W})\right)_{t}$-Brownian motions on the probability space $(\mathbb{W}, \mathcal{B}(\mathbb{W}), \mathbb{P})$. To cater for the two noise terms present in the stochastic MHD equations, we take two copies of $\mathbb{W}$ and take their product $\mathfrak{W}:=\mathbb{W} \times \mathbb{W}$, with the metric

$$
\rho_{\mathfrak{W}}\left((w, \bar{w}),\left(w^{\prime}, \bar{w}^{\prime}\right)\right):=\rho_{\mathbb{W}}\left(w, w^{\prime}\right)+\rho_{\mathbb{W}}\left(\bar{w}, \bar{w}^{\prime}\right)
$$

Then $\left(\mathfrak{W}, \rho_{\mathfrak{W}}\right)$ is also a Polish space. In the same way as above, we introduce the filtration $\mathcal{B}_{t}(\mathfrak{W}) \subset \mathcal{B}(\mathfrak{W})$, and endow $(\mathfrak{W}, \mathcal{B}(\mathfrak{W})$ ) with the product $\mathfrak{P}:=\mathbb{P} \otimes \mathbb{P}$ of the two Wiener measures. Then the coordinate process

$$
\mathcal{W}(t):=\left(\begin{array}{l}
W(t) \\
\bar{W}(t)
\end{array}\right)
$$

consists of two independent infinite sequences of independent standard Brownian motions. For simplicity, in the following we will sometimes refer to the process $\mathcal{W}$ as a Brownian motion.

Now consider the space of continuous, $\mathcal{H}^{1}$-valued paths that are square-integrable in time with values in $\mathcal{H}^{2}, \mathfrak{B}:=C\left(\mathbb{R}_{+} ; \mathcal{H}^{1}\right) \cap L_{\text {loc }}^{2}\left(\mathbb{R}_{+} ; \mathcal{H}^{2}\right)$ with the metric

$$
\rho_{\mathfrak{B}}\left(y, y^{\prime}\right):=\sum_{k \in \mathbb{N}} 2^{-k}\left(\sup _{t \in[0, k]}\left\|y(t)-y^{\prime}(t)\right\|_{\mathcal{H}^{1}}+\int_{0}^{k}\left\|y(t)-y^{\prime}(t)\right\|_{\mathcal{H}^{2}}^{2} \mathrm{~d} t\right) \wedge 1 .
$$

The $\sigma$-algebra up to time $t$ of this space is denoted by $\mathcal{B}_{t}(\mathfrak{B}) \subset \mathcal{B}(\mathfrak{B})$. For any measure space $(S, \mathcal{S}, \lambda)$, we denote the completion of the $\sigma$-algebra $\mathcal{S}$ with respect to the measure $\lambda$ by $\overline{\mathcal{S}}^{\lambda}$.

Definition 3.4 Let $\left(\Omega, \mathcal{F}, P,\left(\mathcal{F}_{t}\right)_{t \geq 0} ; \mathcal{W} ; y\right)$ be a weak solution of Eq. (9) with initial distribution $\vartheta \in \mathcal{P}\left(\mathcal{H}^{1}\right)$. If there exists a ${\overline{\mathcal{B}\left(\mathcal{H}^{1}\right) \times \mathcal{B}(\mathfrak{W})}}^{\vartheta \otimes P} / \mathcal{B}(\mathfrak{B})$-measurable function $F_{\vartheta}: \mathcal{H}^{1} \times \mathfrak{W} \rightarrow \mathfrak{B}$ such that

(i) For every $t>0, F_{\vartheta}$ is $\hat{\mathcal{B}}_{t} / \mathcal{B}_{t}(\mathfrak{B})$-measurable, where $\hat{\mathcal{B}}_{t}:={\overline{\mathcal{B}\left(\mathcal{H}^{1}\right) \times \mathcal{B}_{t}(\mathfrak{W J})}}^{\vartheta \otimes P}$, (ii) $y(\cdot)=F_{\vartheta}(y(0), \mathcal{W}(\cdot)), P$-a.s.,

then we call $(\mathcal{W}, y)$ a strong solution.

Remark 3.5 The function $F_{\vartheta}$ is a "machine" that turns an initial value $y_{0} \in \mathcal{H}^{1}$ and a Brownian motion $\mathcal{W} \in \mathfrak{W}$ into a solution via $y=F_{\vartheta}\left(y_{0}, \mathcal{W}\right)$. The first property of $F_{\vartheta}$ is a type of adaptedness.

Our next definition serves the purpose to clarify what we mean by a unique solution.

Definition 3.6 Equation (9) is said to have a unique strong solution associated to $\vartheta \in \mathcal{P}\left(\mathcal{H}^{1}\right)$ if there exists a function $F_{\vartheta}: \mathcal{H}^{1} \times \mathfrak{W} \rightarrow \mathfrak{B}$ as in Definition 3.4 such that also the following two conditions are satisfied: 
(i) for any two independent copies of infinite sequence of independent standard Brownian motions $\{\mathcal{W}(t) \mid t \geq 0\}$ on the stochastic basis $\left(\Omega, \mathcal{F}, P,\left(\mathcal{F}_{t}\right)_{t \geq 0}\right)$ and any $\mathcal{H}^{1}$-valued, $\mathcal{F}_{0}$-measurable random variable $y_{0}$ with distribution $P \circ y_{0}^{-1}=\vartheta$,

$\left(\Omega, \mathcal{F}, P,\left(\mathcal{F}_{t}\right)_{t \geq 0} ; \mathcal{W} ; F_{\vartheta}\left(y_{0}, \mathcal{W}(\cdot)\right)\right)$ is a weak solution of Equation (9);

(ii) for any weak solution $\left(\Omega, \mathcal{F}, P,\left(\mathcal{F}_{t}\right)_{t \geq 0} ; \mathcal{W} ; y\right)$ of Eq. (9) with initial law $\vartheta$,

$$
y(\cdot)=F_{\vartheta}(y(0), \mathcal{W}(\cdot)), \quad P-a . s .
$$

The following Yamada-Watanabe theorem shows the relationship between the different definitions introduced in this section and gives a way to show existence of a unique strong solution to our equation.

Theorem 3.7 (Yamada-Watanabe) If there exists a weak solution to Eq. (9) and pathwise uniqueness holds, then there exists a unique strong solution to Eq. (9).

Proof This follows by applying the results of Röckner et al. [36, Theorem 2.1].

\subsection{Pathwise uniqueness}

In this section we prove that pathwise uniqueness holds for the stochastic tamed MHD equations, following the ideas of [39].

Theorem 3.8 (Pathwise uniqueness) Let the Assumptions (H1)-(H3) be satisfied. Then pathwise uniqueness holds for (9).

Proof Let $y_{1}, y_{2}$ belong to two weak solutions defined on the same stochastic basis $\left(\Omega, \mathcal{F}, P,\left(\mathcal{F}_{t}\right)_{t \geq 0}\right)$ and with the same Brownian motion $\mathcal{W}$ and same initial condition $y_{0}$. Let $T>0, R>0$ and define the stopping time

$$
\tau_{R}:=\inf \left\{t \in[0, T] \mid\left\|y_{1}(t)\right\|_{\mathcal{H}^{1}} \vee\left\|y_{2}(t)\right\|_{\mathcal{H}^{1}} \geq R\right\} \wedge T
$$

By Assumption (ii) of the definition of a weak solution, we know that for $P$-a.e. $\omega$ $y_{i}(\cdot, \omega) \in C\left([0, T] ; \mathcal{H}^{1}\right), i=1,2$, and thus

$$
\tau_{R} \uparrow T, \quad \text { as } R \rightarrow \infty, \quad P-\text { a.s. }
$$

So $\tau_{R}$ is a localising sequence. Now we set $z(t):=y_{1}(t)-y_{2}(t)$. $z$ satisfies the equation

$$
\begin{aligned}
\mathrm{d} z(t)= & {\left[\mathcal{A}\left(y_{1}(t)\right)-\mathcal{A}\left(y_{2}(t)\right)+\mathcal{P}\left(f\left(t, y_{1}(t)\right)-f\left(t, y_{2}(t)\right)\right)\right] \mathrm{d} t } \\
& +\sum_{k=1}^{\infty}\left(\mathcal{B}_{k}\left(t, y_{1}(t)\right)-\mathcal{B}_{k}\left(t, y_{2}(t)\right)\right) \mathrm{d} \mathcal{W}_{t}^{k}, \\
z(0)= & 0 .
\end{aligned}
$$


Thus by Itô's formula for $\|z\|_{\mathcal{H}^{0}}^{2}$, we find (noting the self-adjointness of the projection $\mathcal{P}$ with respect to $\left.\langle\cdot, \cdot\rangle_{\mathcal{H}^{0}}=\langle\cdot, \cdot\rangle_{\mathcal{L}^{2}}\right)$

$$
\begin{aligned}
\|z(t)\|_{\mathcal{H}^{0}}^{2}= & 2 \int_{0}^{t}\left\langle\mathcal{A}\left(y_{1}(s)\right)-\mathcal{A}\left(y_{2}(s)\right), z(s)\right\rangle_{\mathcal{H}^{0}} \mathrm{~d} s \\
& +2 \int_{0}^{t}\left\langle f\left(s, y_{1}(s)\right)-f\left(s, y_{2}(s)\right), z(s)\right\rangle_{\mathcal{H}^{0}} \mathrm{~d} s \\
& +2 \sum_{k=1}^{\infty} \int_{0}^{t}\left\langle\mathcal{B}_{k}\left(s, y_{1}(s)\right)-\mathcal{B}_{k}\left(s, y_{2}(s)\right), z(s)\right\rangle_{\mathcal{H}^{0}} \mathrm{~d} \mathcal{W}_{s}^{k} \\
& +\sum_{k=1}^{\infty} \int_{0}^{t}\left\|\mathcal{B}_{k}\left(s, y_{1}(s)\right)-\mathcal{B}_{k}\left(s, y_{2}(s)\right)\right\|_{\mathcal{H}^{0}}^{2} \mathrm{~d} s \\
= & : I_{1}(t)+I_{2}(t)+I_{3}(t)+I_{4}(t) .
\end{aligned}
$$

We stop with $\tau_{R}$ and estimate each term separately.

The first term $I_{1}\left(t \wedge \tau_{R}\right)$ can be rewritten and estimated using integration by parts and Young's inequality:

$$
\begin{aligned}
& I_{1}\left(t \wedge \tau_{R}\right) \leq-\int_{0}^{t \wedge \tau_{R}}\|\nabla z(s)\|_{\mathcal{H}^{0}}^{2} \mathrm{~d} s \\
& +2 \int_{0}^{t \wedge \tau_{R}}\left[\left\|\boldsymbol{v}_{1} \otimes \boldsymbol{v}_{1}-\boldsymbol{v}_{2} \otimes \boldsymbol{v}_{2}\right\|_{\mathbb{L}^{2}}^{2}+\left\|\boldsymbol{B}_{1} \otimes \boldsymbol{B}_{1}-\boldsymbol{B}_{2} \otimes \boldsymbol{B}_{2}\right\|_{\mathbb{L}^{2}}^{2}\right. \\
& \left.+\left\|\boldsymbol{v}_{1} \otimes \boldsymbol{B}_{1}-\boldsymbol{v}_{2} \otimes \boldsymbol{B}_{2}\right\|_{\mathbb{L}^{2}}^{2}+\left\|\boldsymbol{B}_{1} \otimes \boldsymbol{v}_{1}-\boldsymbol{B}_{2} \otimes \boldsymbol{v}_{2}\right\|_{\mathbb{L}^{2}}^{2}\right] \mathrm{d} s \\
& \quad-2 \int_{0}^{t \wedge \tau_{R}}\left\langle g_{N}\left(y_{1}\right) y_{1}-g_{N}\left(y_{2}\right) y_{2}, z\right\rangle_{\mathcal{L}^{2}} \mathrm{~d} s=: J_{1}\left(t \wedge \tau_{R}\right)+J_{2}\left(t \wedge \tau_{R}\right)+J_{3}\left(t \wedge \tau_{R}\right) .
\end{aligned}
$$

The terms of $J_{2}\left(t \wedge \tau_{R}\right)$ are of the general form

$$
\left\|\boldsymbol{\theta}_{1} \otimes \boldsymbol{\psi}_{1}-\boldsymbol{\theta}_{2} \otimes \boldsymbol{\psi}_{2}\right\|_{\mathbb{L}^{2}}^{2}, \quad \boldsymbol{\theta}_{i}, \boldsymbol{\psi}_{i} \in\{\boldsymbol{v}, \boldsymbol{B}\}, i=1,2
$$

and can be estimated as follows:

$$
\begin{aligned}
\| \boldsymbol{\theta}_{1} & \otimes \boldsymbol{\psi}_{1}-\boldsymbol{\theta}_{2} \otimes \boldsymbol{\psi}_{2} \|_{\mathbb{L}^{2}}^{2} \\
& \leq 2\left(\left\|\boldsymbol{\theta}_{1}\right\|_{\mathbb{L}^{4}}^{2}\left\|\boldsymbol{\psi}_{1}-\boldsymbol{\psi}_{2}\right\|_{\mathbb{L}^{4}}^{2}+\left\|\boldsymbol{\theta}_{1}-\boldsymbol{\theta}_{2}\right\|_{\mathbb{L}^{4}}^{2}\left\|\boldsymbol{\psi}_{2}\right\|_{\mathbb{L}^{4}}^{2}\right) \\
& \leq 2 C_{1,4}^{2}\left(\left\|\boldsymbol{\theta}_{1}\right\|_{\mathbb{H}^{1}}^{2}\left\|\boldsymbol{\psi}_{1}-\boldsymbol{\psi}_{2}\right\|_{\mathbb{H}^{1}}^{3 / 2}\left\|\boldsymbol{\psi}_{1}-\boldsymbol{\psi}_{2}\right\|_{\mathbb{H}^{0}}^{1 / 2}+\left\|\boldsymbol{\theta}_{1}-\boldsymbol{\theta}_{2}\right\|_{\mathbb{H}^{1}}^{3 / 2}\left\|\boldsymbol{\theta}_{1}-\boldsymbol{\theta}_{2}\right\|_{\mathbb{H}^{0}}^{1 / 2}\left\|\boldsymbol{\psi}_{2}\right\|_{\mathbb{H}^{1}}^{2}\right) \\
& \leq 2 C_{1,4}^{2} R^{2}\left(\left\|\boldsymbol{\psi}_{1}-\boldsymbol{\psi}_{2}\right\|_{\mathbb{H}^{1}}^{3 / 2}\left\|\boldsymbol{\psi}_{1}-\boldsymbol{\psi}_{2}\right\|_{\mathbb{H}^{0}}^{1 / 2}+\left\|\boldsymbol{\theta}_{1}-\boldsymbol{\theta}_{2}\right\|_{\mathbb{H}^{1}}^{3 / 2}\left\|\boldsymbol{\theta}_{1}-\boldsymbol{\theta}_{2}\right\|_{\mathbb{H}^{0}}^{1 / 2}\right) \\
& \leq \frac{1}{16}\left\|\boldsymbol{\psi}_{1}-\boldsymbol{\psi}_{2}\right\|_{\mathbb{H}^{1}}^{2}+\frac{1}{16}\left\|\boldsymbol{\theta}_{1}-\boldsymbol{\theta}_{2}\right\|_{\mathbb{H}^{1}}^{2}+C_{R}\left(\left\|\boldsymbol{\psi}_{1}-\boldsymbol{\psi}_{2}\right\|_{\mathbb{H}^{0}}^{2}+\left\|\boldsymbol{\theta}_{1}-\boldsymbol{\theta}_{2}\right\|_{\mathbb{H}^{0}}^{2}\right) .
\end{aligned}
$$


Counting all possible combinations of $\boldsymbol{\theta}$ and $\boldsymbol{\psi}$ and combining the $\boldsymbol{v}$ and $\boldsymbol{B}$-norms into the corresponding norms for $y$, we find that

$$
J_{2}\left(t \wedge \tau_{R}\right) \leq \frac{1}{4} \int_{0}^{t}\|\nabla z(s)\|_{\mathcal{H}^{0}}^{2} \mathrm{~d} s+C_{R} \int_{0}^{t}\|z(s)\|_{\mathcal{H}^{0}}^{2} \mathrm{~d} s .
$$

Since $\left|g_{N}(r)-g_{N}\left(r^{\prime}\right)\right| \leq 2\left|r-r^{\prime}\right|$, we see, using a short calculation as well as Sobolev embedding and Young's inequality, that

$$
\begin{aligned}
J_{3}\left(t \wedge \tau_{R}\right) & \leq 8 \int_{0}^{t \wedge \tau_{R}}\left\||z| \cdot\left(\left|y_{1}\right|+\left|y_{2}\right|\right)\right\|_{L^{2}}^{2} \mathrm{~d} s \leq 16 \int_{0}^{t \wedge \tau_{R}}\|z\|_{\mathcal{L}^{4}}^{2}\left(\left\|y_{1}\right\|_{\mathcal{L}^{4}}^{2}+\left\|y_{2}\right\|_{\mathcal{L}^{4}}^{2}\right) \mathrm{d} s \\
& \leq 16 C_{1,4}^{2} \int_{0}^{t \wedge \tau_{R}}\|\nabla z\|_{\mathcal{H}^{0}}^{3 / 2}\|z\|_{\mathcal{H}^{0}}^{1 / 2}\left(\left\|y_{1}\right\|_{\mathcal{H}^{1}}^{2}+\left\|y_{2}\right\|_{\mathcal{H}^{1}}^{2}\right) \mathrm{d} s \\
& \leq 16 C_{1,4}^{2} R^{2} \int_{0}^{t \wedge \tau_{R}}\|\nabla z\|_{\mathcal{H}^{0}}^{3 / 2}\|z\|_{\mathcal{H}^{0}}^{1 / 2} \mathrm{~d} s \\
& \leq \frac{1}{4} \int_{0}^{t \wedge \tau_{R}}\|\nabla z\|_{\mathcal{H}^{0}}^{2} \mathrm{~d} s+C_{R} \int_{0}^{t \wedge \tau_{R}}\|z\|_{\mathcal{H}^{0}}^{2} \mathrm{~d} s .
\end{aligned}
$$

Thus, altogether we find that

$$
I_{1}\left(t \wedge \tau_{R}\right) \leq-\frac{1}{2} \int_{0}^{t \wedge \tau_{R}}\|\nabla z(s)\|_{\mathcal{H}^{0}}^{2} \mathrm{~d} s+C_{R} \int_{0}^{t \wedge \tau_{R}}\|z(s)\|_{\mathcal{H}^{0}}^{2} \mathrm{~d} s
$$

By Cauchy-Schwarz-Buniakowski and Assumption (H1), we find for $I_{2}\left(t \wedge \tau_{R}\right)$

$$
2 \int_{0}^{t}\left\langle f\left(s, y_{1}(s)\right)-f\left(s, y_{2}(s)\right), z(s)\right\rangle_{\mathcal{H}^{0}} \mathrm{~d} s \leq C_{T, F} \int_{0}^{t \wedge \tau_{R}}\|z(s)\|_{\mathcal{H}^{0}}^{2} \mathrm{~d} s
$$

The term $I_{3}\left(t \wedge \tau_{R}\right)$ is a martingale and thus killed upon taking expectations.

For $I_{4}\left(t \wedge \tau_{R}\right)$ we have by Assumptions (H2) and (H3)

$$
\begin{aligned}
& I_{4}\left(t \wedge \tau_{R}\right)=\sum_{k=1}^{\infty} \int_{0}^{t \wedge \tau_{R}}\left\|\mathcal{P}\left(\left(\Sigma^{k} \cdot \nabla\right) z(s)\right)+\mathcal{P}\left(H^{k}\left(s, y_{1}(s)\right)-H^{k}\left(s, y_{2}(s)\right)\right)\right\|_{\mathcal{H}^{0}}^{2} \mathrm{~d} s \\
& \quad \leq \sup _{t \in[0, T], x \in \mathbb{D}}\|\Sigma(t, x)\|_{\ell^{2}}^{2} \int_{0}^{t \wedge \tau_{R}}\|\nabla z(s)\|_{\mathcal{H}^{0}}^{2} \mathrm{~d} s+C_{T, H} \int_{0}^{t \wedge \tau_{R}}\|z(s)\|_{\mathcal{H}^{0}}^{2} \mathrm{~d} s \\
& \quad \leq \frac{1}{4} \int_{0}^{t \wedge \tau_{R}}\|\nabla z(s)\|_{\mathcal{H}^{0}}^{2} \mathrm{~d} s+C_{T, H} \int_{0}^{t \wedge \tau_{R}}\|z(s)\|_{\mathcal{H}^{0}}^{2} \mathrm{~d} s .
\end{aligned}
$$


Hence, if we stop (21) with $\tau_{R}$ and take expectations, we find, using the previous estimates, that

$$
\mathbb{E}\left[\left\|z\left(t \wedge \tau_{R}\right)\right\|_{\mathcal{H}^{0}}^{2}\right] \leq C_{R, T} \mathbb{E}\left[\int_{0}^{t \wedge \tau_{R}}\|z(s)\|_{\mathcal{H}^{0}}^{2} \mathrm{~d} s\right] \leq C_{R, T, f, H} \int_{0}^{t} \mathbb{E}\left[\left\|z\left(s \wedge \tau_{R}\right)\right\|_{\mathcal{H}^{0}}^{2}\right] \mathrm{d} s .
$$

Applying Gronwall's lemma yields that for any $t \in[0, T]$

$$
\mathbb{E}\left[\left\|z\left(t \wedge \tau_{R}\right)\right\|_{\mathcal{H}^{0}}^{2}\right]=0
$$

Finally, we employ Fatou's lemma to find

$$
\mathbb{E}\left[\|z(t)\|_{\mathcal{H}^{0}}^{2}\right] \leq \liminf _{R \rightarrow \infty} \mathbb{E}\left[\left\|z\left(t \wedge \tau_{R}\right)\right\|_{\mathcal{H}^{0}}^{2}\right]=0
$$

Thus, $z(t)=0$ for all $t \geq 0, P$-a.s., i.e., pathwise uniqueness holds.

\subsection{Existence of martingale solutions}

The main result of this section is the following:

Theorem 3.9 Under the Assumptions (H1)-(H3), for any initial law $\vartheta \in \mathcal{P}\left(\mathcal{H}^{1}\right)$, there exists a weak solution for Eq. (9) in the sense of Definition 3.1.

The proof proceeds in the usual fashion, analogously to the proof of [39, Theorem 3.8] by considering Faedo-Galerkin approximations of our equation and will be carried out at the end of this section. To be more precise, fix a stochastic basis $\left(\Omega, \mathcal{F}, P,\left(\mathcal{F}_{t}\right)_{t \geq 0}\right)$ and two independent infinite sequences of independent standard $\left(\mathcal{F}_{t}\right)_{t}$-Brownian motions $\left\{\mathcal{W}^{k}(t) \mid t \geq 0, k \in \mathbb{N}\right\}$ and an $\mathcal{F}_{0}$-measurable random variable $y_{0}$ with initial law $\vartheta \in \mathcal{P}\left(\mathcal{H}^{1}\right)$.

The set $\mathcal{E}=\left\{e_{i} \mid i \in \mathbb{N}\right\} \subset \mathcal{V}$ was chosen as a complete orthonormal basis of $\mathcal{H}^{1}$. We consider the finite-dimensional subspaces

$$
\mathcal{H}_{n}^{1}:=\operatorname{span}\left\{e_{i} \mid i=1, \ldots, n\right\}
$$

and consider the projections onto $\mathcal{H}_{n}^{1}$, i.e. for $y \in \mathcal{H}^{0}$, we define (recalling our convention (19))

$$
\Pi_{n} y:=\sum_{i=1}^{n}\left\langle y, e_{i}\right\rangle_{\mathcal{H}^{1}} e_{i}=\sum_{i=1}^{n}\left\langle y,(I-\Delta) e_{i}\right\rangle_{\mathcal{H}^{0}} e_{i}
$$


We want to study the following finite-dimensional stochastic ordinary differential equations in $\mathcal{H}_{n}^{1}$ as approximations for our infinite-dimensional equation:

$$
\left\{\begin{array}{l}
\mathrm{d} y_{n}(t)=\left[\Pi_{n} \mathcal{A}\left(y_{n}(t)\right)+\Pi_{n} f\left(t, y_{n}(t)\right)\right] \mathrm{d} t+\sum_{k} \Pi_{n} \mathcal{B}_{k}\left(t, y_{n}(t)\right) \mathrm{d} \mathcal{W}_{t}^{k} \\
y_{n}(0)=\Pi_{n} y_{0}
\end{array}\right.
$$

Using the Lemmas 2.5 and 2.6, there exists a constant $C_{n, N}$ such that for any $y \in \mathcal{H}_{n}^{1}$ the following growth conditions hold:

$$
\begin{aligned}
\left\langle y, \Pi_{n} \mathcal{A}(y)+\Pi_{n} f(t, y)\right\rangle_{\mathcal{H}_{n}^{1}} & \leq C_{n, N}\left(\|y\|_{\mathcal{H}_{n}^{1}}^{2}+1\right), \\
\left\|\Pi_{n} \mathcal{B}(t, y)\right\|_{L_{2}\left(\ell^{2} \times \ell^{2} ; \mathcal{H}_{n}^{1}\right)} & \leq C_{n, N}\left(\|y\|_{\mathcal{H}_{n}^{1}}^{2}+1\right) .
\end{aligned}
$$

Therefore, we can employ the theory of stochastic (ordinary) differential equations (cf. [26, Theorem 3.1.1, p. 56]) to find a unique $\left(\mathcal{F}_{t}\right)_{t}$-adapted process $y_{n}(t)$ such that $P$-a.s. for all $t \in[0, T]$

$$
\begin{aligned}
y_{n}(t)= & y_{n}(0)+\int_{0}^{t} \Pi_{n} \mathcal{A}\left(y_{n}(s)\right) \mathrm{d} s+\int_{0}^{t} \Pi_{n} f\left(s, y_{n}(s)\right) \mathrm{d} s \\
& +\sum_{k=1}^{\infty} \int_{0}^{t} \Pi_{n} \mathcal{B}_{k}\left(s, y_{n}(s)\right) \mathrm{d} \mathcal{W}_{s}^{k},
\end{aligned}
$$

and for any $i \leq n$

$$
\begin{aligned}
\left\langle y_{n}(t), e_{i}\right\rangle_{\mathcal{H}^{1}}= & \left\langle y_{n}(0), e_{i}\right\rangle_{\mathcal{H}^{1}}+\int_{0}^{t}\left\langle\mathcal{A}\left(y_{n}(s)\right), e_{i}\right\rangle_{\mathcal{H}^{1}} \mathrm{~d} s+\int_{0}^{t}\left\langle f\left(s, y_{n}(s)\right), e_{i}\right\rangle_{\mathcal{H}^{1}} \mathrm{~d} s \\
& +\sum_{k=1}^{\infty} \int_{0}^{t}\left\langle\mathcal{B}_{k}\left(s, y_{n}(s)\right), e_{i}\right\rangle_{\mathcal{H}^{1}} \mathrm{~d} \mathcal{W}_{s}^{k} .
\end{aligned}
$$

Our strategy now is as follows:

1. Prove uniform a priori estimates for $y_{n}$.

2. Use these to prove tightness of the associated laws.

3. Use Skorokhod's embedding theorem to translate the weak convergence from the previous step to $P$-a.s. convergence of the random variables.

4. Prove uniform moment estimates for the terms of the associated martingale problem.

5. Show convergence in probability of the martingale problems.

We start with the a priori estimates. 
Lemma 3.10 (A priori estimates) For any $T>0$, there exists a constant $C_{T, N, f, H, y_{0}}>$ 0 such that for any $n \in \mathbb{N}$

$$
\begin{aligned}
& \mathbb{E}\left[\sup _{t \in[0, T]}\left\|y_{n}(t)\right\|_{\mathcal{H}^{1}}^{2}\right]+\int_{0}^{T} \mathbb{E}\left[\left\|y_{n}(s)\right\|_{\mathcal{H}^{2}}^{2}\right] \mathrm{d} s+\int_{0}^{T} \mathbb{E}\left[\left\|\nabla\left|y_{n}(s)\right|^{2}\right\|_{L^{2}}^{2}\right] \mathrm{d} s \\
& \quad \leq C_{T, N, f, H, y_{0} .}
\end{aligned}
$$

Furthermore, in the periodic case it holds that

$$
\int_{0}^{t} \mathbb{E}\left[\left\|y_{n}(s)\right\|_{\mathcal{L}^{4}}^{4}\right] \mathrm{d} s \leq C_{T, N, f, H, y_{0}} .
$$

Proof The proof of this proceeds in a standard way: we use Itô's formula for $\left\|y_{n}(t)\right\|_{\mathcal{H}^{1}}^{2}$ to find

$$
\begin{aligned}
\left\|y_{n}(t)\right\|_{\mathcal{H}^{1}}^{2}= & \left\|y_{0}\right\|_{\mathcal{H}^{1}}^{2}+2 \int_{0}^{t}\left\langle\mathcal{A}\left(y_{n}(s)\right), y_{n}(s)\right\rangle_{\mathcal{H}^{1}} \mathrm{~d} s+2 \int_{0}^{t}\left\langle f\left(s, y_{n}(s)\right), y_{n}(s)\right\rangle_{\mathcal{H}^{1}} \mathrm{~d} s \\
& +M(t)+\int_{0}^{t}\left\|\mathcal{B}\left(s, y_{n}(s)\right)\right\|_{L_{2}\left(\ell^{2} ; \mathcal{H}^{1}\right)}^{2} \mathrm{~d} s
\end{aligned}
$$

where the term $M(t)$ is a continuous martingale and has the representation

$$
M(t):=2 \sum_{k=1}^{\infty}\left\langle\mathcal{B}_{k}\left(s, y_{n}(s)\right), y_{n}(s)\right\rangle_{\mathcal{H}^{1}} \mathrm{~d} \mathcal{W}_{s}^{k}
$$

Then we use Lemmas 2.4 and 2.6 to estimate the terms on the right-hand side, take expectation and apply Young's inequality and Gronwall's lemma to arrive at an inequality of the form

$$
\begin{aligned}
& \sup _{t \in[0, T]} \mathbb{E}\left\|y_{n}(t)\right\|_{\mathcal{H}^{1}}^{2}+\int_{0}^{T}\left\|y_{n}(s)\right\|_{\mathcal{H}^{2}}^{2} \mathrm{~d} s+\int_{0}^{T} \mathbb{E}\left[\left\|\left|\boldsymbol{v}_{n}\right| \cdot\left|\nabla \boldsymbol{v}_{n}\right|\right\|_{L^{2}}^{2}\right. \\
& \left.\quad+\left\|\left|\boldsymbol{B}_{n}\right| \cdot\left|\nabla \boldsymbol{B}_{n}\right|\right\|_{L^{2}}^{2}+\left\|\left|\boldsymbol{v}_{n}\right| \cdot\left|\nabla \boldsymbol{B}_{n}\right|\right\|_{L^{2}}^{2}+\left\|\left|\boldsymbol{B}_{n}\right| \cdot\left|\nabla \boldsymbol{v}_{n}\right|\right\|_{L^{2}}^{2}\right] \mathrm{d} s \\
& \leq C_{T, N, H, f, y_{0} .}
\end{aligned}
$$

To "exchange" expectation and supremum, we start again from (26), take suprema first and then expectations, and then use the Burkholder-Davis-Gundy (BDG) inequality (cf. [29], Theorem 1.1) and Young's inequality. Lemma 2.6 as well as the estimate (27) just obtained yield the claimed estimate.

In the periodic case, as $\mathcal{E}$ is also orthogonal in $\mathcal{H}^{0}$, so we get an Itô formula for the $\mathcal{H}^{0}$-norms as well and conclude taking expectations and using (12) of Lemma 2.4 as well as Eq. (24). Gronwall's lemma then implies (25).

Next we want to prove tightness of the laws of the solutions to the approximate equations (22). We recall the notation $\mathcal{X}:=C\left(\mathbb{R}_{+} ; \mathcal{H}_{\text {loc }}^{0}\right)$ from Sect. 2.3. 
Lemma 3.11 (Tightness) Let $\mu_{n}:=P \circ\left(y_{n}\right)^{-1}$ be the law of $y_{n}$ in $(\mathcal{X}, \mathcal{B}(\mathcal{X}))$. Then the family $\left(\mu_{n}\right)_{n \in \mathbb{N}}$ is tight on $(\mathcal{X}, \mathcal{B}(\mathcal{X}))$.

Proof Let $R>0$. We set

$$
\tau_{R}^{n}:=\inf \left\{t \geq 0 \mid\left\|y_{n}(t)\right\|_{\mathcal{H}_{n}^{1}} \geq R\right\}
$$

Then, using the Chebychev inequality as well as the a priori estimate (24), we find

$$
\begin{aligned}
\sup _{n \in \mathbb{N}} P\left(\tau_{R}^{n}<T\right) & =\sup _{n \in \mathbb{N}} P\left(\sup _{t \in[0, T]}\left\|y_{n}(t)\right\|_{\mathcal{H}_{n}^{1}} \geq R\right) \\
& \leq \sup _{n \in \mathbb{N}} \frac{1}{R^{2}} \mathbb{E}\left[\sup _{t \in[0, T]}\left\|y_{n}(t)\right\|_{\mathcal{H}_{n}^{1}}^{2}\right] \leq \frac{C_{T, N, f, H, y_{0}}}{R^{2}} .
\end{aligned}
$$

For any $q \geq 2$ and $s, t \in[0, T]$ and any $e \in \mathcal{E}$ (whose support we denote by $\mathcal{O}$ ), we find by using Equations (23), (14) as well as the BDG inequality (cf. [29], Theorem 1.1)

$$
\begin{aligned}
\mathbb{E} & {\left[\left|\left\langle y_{n}\left(t \wedge \tau_{R}^{n}\right)-y_{n}\left(s \wedge \tau_{R}^{n}\right), e\right\rangle_{\mathcal{H}^{1}}\right|^{q}\right] } \\
\leq & 3^{q-1}\left(\mathbb{E}\left[\left|\int_{s \wedge \tau_{R}^{n}}^{t \wedge \tau_{R}^{n}}\left\langle\mathcal{A}\left(y_{n}(r)\right), e\right\rangle_{\mathcal{H}^{1}} \mathrm{~d} r\right|^{q}\right]+\mathbb{E}\left[\left|\int_{s \wedge \tau_{R}^{n}}^{t \wedge \tau_{R}^{n}}\left\langle f\left(r, y_{n}(r)\right), e\right\rangle_{\mathcal{H}^{1}} \mathrm{~d} r\right|^{q}\right]\right. \\
& \left.+\mathbb{E}\left[\left|\sum_{k=1}^{\infty} \int_{s \wedge \tau_{R}^{n}}^{t \wedge \tau_{R}^{n}}\left\langle\mathcal{B}_{k}\left(r, y_{n}(r)\right), e\right\rangle_{\mathcal{H}^{1}} \mathrm{~d} \mathcal{W}_{r}^{k}\right|^{q}\right]\right) \\
\leq & C_{e, q}\left(\mathbb{E}\left[\left|\int_{s \wedge \tau_{R}^{n}}^{t \wedge \tau_{R}^{n}}\left(1+\left\|y_{n}\right\|_{\mathcal{L}^{3}(\mathcal{O})}^{3}\right) \mathrm{d} r\right|^{q}\right]+\mathbb{E}\left[\left|\int_{s \wedge \tau_{R}^{n}}^{t \wedge \tau_{R}^{n}}\left\|f\left(r, y_{n}(r)\right)\right\|_{\mathcal{H}^{0}} \mathrm{~d} r\right|^{q}\right]\right. \\
& \left.+\mathbb{E}\left[\left|\int_{s \wedge \tau_{R}^{n}}^{t \wedge \tau_{R}^{n}}\left\|\mathcal{B}\left(r, y_{n}(r)\right)\right\|_{L_{2}\left(\ell^{2} \times \ell^{2} ; \mathcal{H}^{0}\right)}^{2} \mathrm{~d} r\right|^{q / 2}\right]\right),
\end{aligned}
$$

where we transferred all the spatial derivatives onto $e$ via (19). Applying the Hölder embedding $\mathcal{L}^{6}(\mathcal{O}) \subset \mathcal{L}^{3}(\mathcal{O})$ as well as the Sobolev embedding $\mathcal{H}^{1} \subset \mathcal{L}^{6}$, Assumptions (H1), (H3) and Eq. (17), we can see that

$$
\begin{aligned}
\mathbb{E}\left[\left|\left\langle y_{n}\left(t \wedge \tau_{R}^{n}\right)-y_{n}\left(s \wedge \tau_{R}^{n}\right), e\right\rangle_{\mathcal{H}^{1}}\right|^{q}\right] \\
\leq C_{e, q, T}\left(\mathbb{E}\left[\left|\int_{s \wedge \tau_{R}^{n}}^{t \wedge \tau_{R}^{n}}\left(1+\sup _{r \in[0, T]}\left\|y_{n}(r)\right\|_{\mathcal{H}^{1}}^{3}\right) \mathrm{d} r\right|^{q}\right]\right. \\
+\mathbb{E}\left[\left|\int_{s \wedge \tau_{R}^{n}}^{t \wedge \tau_{R}^{n}}\left\|y_{n}(r)\right\|_{\mathcal{H}^{1}}^{2}+\left\|F_{f}(r)\right\|_{L^{1}(\mathbb{D})} \mathrm{d} r\right|^{q}\right] \\
\left.+\mathbb{E}\left[\left|\int_{s \wedge \tau_{R}^{n}}^{t \wedge \tau_{R}^{n}}\left\|y_{n}(r)\right\|_{\mathcal{H}^{1}}^{2}+\left\|F_{H}(r)\right\|_{L^{1}(\mathbb{D})} \mathrm{d} r\right|^{q / 2}\right]\right)
\end{aligned}
$$




$$
\begin{aligned}
\leq & C_{e, q, T}\left\{\mathbb{E}\left[\left(1+\sup _{r \in\left[0, T \wedge \tau_{R}^{n}\right]}\left\|y_{n}(r)\right\|_{\mathcal{H}^{1}}^{3 q}\right)\right]|t-s|^{q}\right. \\
& +\mathbb{E}\left[\left(\sup _{r \in\left[0, T \wedge \tau_{R}^{n}\right]}\left\|y_{n}(r)\right\|_{\mathcal{H}^{1}}^{2 q}+\left\|F_{f}\right\|_{L^{\infty}\left([0, T] ; L^{1}(\mathbb{D})\right)}^{q}\right)\right]|t-s|^{q} \\
& \left.+\mathbb{E}\left[\left(\sup _{r \in\left[0, T \wedge \tau_{R}^{n}\right]}\left\|y_{n}(r)\right\|_{\mathcal{H}^{1}}^{q}+\left\|F_{H}\right\|_{L^{\infty}\left([0, T] ; L^{1}(\mathbb{D})\right)}^{q / 2}\right)\right]|t-s|^{q / 2}\right\} \\
\leq & C_{e, q, T, R} \mathbb{E}\left[\left(1+\sup _{r \in[0, T]}\left\|y_{n}(r)\right\|_{\mathcal{H}^{1}}^{2}+\left\|F_{f}\right\|_{L^{\infty}\left([0, T] ; L^{1}(\mathbb{D})\right)}^{q}\right.\right. \\
& \left.\left.+\left\|F_{H}\right\|_{L^{\infty}\left([0, T] ; L^{1}(\mathbb{D})\right)}^{q / 2}\right)\right]|t-s|^{q / 2} \\
\leq & C_{e, q, T, R, f, H, N, y_{0}|t-s|^{q / 2},}
\end{aligned}
$$

where we have used in the penultimate step that $q \geq 2$ as well as the definition of $\tau_{R}^{n}$ and hence that $\left\|y_{n}(r)\right\|_{\mathcal{H}^{1}}^{q}=\left\|y_{n}(r)\right\|_{\mathcal{H}^{1}}^{q-2} \cdot\left\|y_{n}(r)\right\|_{\mathcal{H}^{1}}^{2} \leq R^{q-2}\left\|y_{n}(r)\right\|_{\mathcal{H}^{1}}^{2}$ and similarly for the other terms. Finally, we have used the a priori estimate (24) in the last step.

Thus by the Kolmogorov-Čentsov continuity criterion (e.g. in the version of [14, Theorem 3.1, p. $28 \mathrm{f}$.]) with parameters $\beta=\frac{q / 2}{q}=1 / 2$, we find that for every $T>0$ and $\alpha \in\left(0, \frac{1}{2}-\frac{1}{q}\right)$

$$
\mathbb{E}\left[\sup _{s, t \in[0, T],|t-s| \leq \delta}\left|\left\langle y_{n}\left(t \wedge \tau_{R}^{n}\right)-y_{n}\left(s \wedge \tau_{R}^{n}\right), e\right\rangle_{\mathcal{H}^{1}}\right|^{q}\right] \leq C_{e, q, T, R, f, H, N, y_{0}} \cdot \delta^{\alpha} .
$$

Therefore, for arbitrary $\varepsilon>0$ and $R>0$, we find using (28)

$$
\begin{aligned}
& \sup _{n \in \mathbb{N}} P\left\{\sup _{s, t \in[0, T],|t-s| \leq \delta}\left|\left\langle y_{n}(t)-y_{n}(s), e\right\rangle_{\mathcal{H}^{1}}\right|>\varepsilon\right\} \\
& \leq \sup _{n \in \mathbb{N}} P\left\{\sup _{s, t \in[0, T],|t-s| \leq \delta}\left|\left\langle y_{n}(t)-y_{n}(s), e\right\rangle_{\mathcal{H}^{1}}\right|>\varepsilon ; \tau_{R}^{n} \geq T\right\}+\sup _{n \in \mathbb{N}} P\left\{\tau_{R}^{n}<T\right\} \\
& \leq \frac{C_{e, q, T, R, f, H, N, y_{0}} \cdot \delta^{\alpha}}{\varepsilon^{q}}+\frac{C_{T, N}}{R^{2}} .
\end{aligned}
$$

Letting first $\delta \downarrow 0$ and then $R \rightarrow \infty$, we find

$$
\lim _{\delta \downarrow 0} \sup _{n \in \mathbb{N}} P\left\{\sup _{s, t \in[0, T],|t-s| \leq \delta}\left|\left\langle y_{n}(t)-y_{n}(s), e\right\rangle_{\mathcal{H}^{1}}\right|>\varepsilon\right\}=0 .
$$

Thanks to (28) and (29), we can now invoke Lemma 2.7 to conclude that $\left(\mu_{n}\right)_{n \in \mathbb{N}}$ is a tight family of probability measures on $(\mathcal{X}, \mathcal{B}(\mathcal{X}))$. 
The tightness implies the existence of a subsequence (which we again denote by $\left.\left(\mu_{n}\right)_{n \in \mathbb{N}}\right)$ that converges weakly to a measure $\mu \in \mathcal{P}(\mathcal{X})$.

Next we apply Skorokhod's coupling theorem (cf. [22, Theorem 4.30, p. 79]) to infer the existence of a probability space $(\tilde{\Omega}, \tilde{\mathcal{F}}, \tilde{P})$ and $\mathcal{X}$-valued random variables $\tilde{y}^{n}$ and $\tilde{y}$ such that

(I) the law of $\tilde{y}^{n}$ is the same as that of $y_{n}$ for all $n \in \mathbb{N}$, i.e. $\tilde{P} \circ \tilde{y}_{n}^{-1}=\mu_{n}$;

(II) the convergence $\tilde{y}_{n} \rightarrow \tilde{y}$ holds in $\mathcal{X}, \tilde{P}$-a.s., and $\tilde{y}$ has law $\mu$.

By Fatou's lemma and the uniform (in $n$ ) a priori estimates (24), the same estimates also hold for the limiting process: for every $T>0$

$$
\mathbb{E}^{\tilde{P}}\left[\sup _{t \in[0, T]}\|\tilde{y}(t)\|_{\mathcal{H}^{1}}^{2}\right] \leq \liminf _{n \rightarrow \infty} \mathbb{E}^{\tilde{P}}\left[\sup _{t \in[0, T]}\left\|\tilde{y}_{n}(t)\right\|_{\mathcal{H}^{1}}^{2}\right]<\infty
$$

as well as

$$
\int_{0}^{T} \mathbb{E}^{\tilde{P}}\left[\|\tilde{y}(s)\|_{\mathcal{H}^{2}}^{2}\right] \mathrm{d} s<\infty
$$

and

$$
\int_{0}^{T} \mathbb{E}^{\tilde{P}}\left[\||\tilde{\boldsymbol{v}}| \cdot|\nabla \tilde{\boldsymbol{v}}|\|_{L^{2}}^{2}+\||\tilde{\boldsymbol{B}}| \cdot|\nabla \tilde{\boldsymbol{B}}|\|_{L^{2}}^{2}+\||\tilde{\boldsymbol{v}}| \cdot|\nabla \tilde{\boldsymbol{B}}|\|_{L^{2}}^{2}+\||\tilde{\boldsymbol{B}}| \cdot|\nabla \tilde{\boldsymbol{v}}|\|_{L^{2}}^{2}\right] \mathrm{d} s<\infty .
$$

In the periodic case $\mathbb{D}=\mathbb{T}^{3}$, we additionally have

$$
\int_{0}^{t} \mathbb{E}^{\tilde{P}}\left[\|\tilde{y}(s)\|_{\mathcal{L}^{4}}^{4}\right] \mathrm{d} s \leq \liminf _{n \rightarrow \infty} \int_{0}^{t} \mathbb{E}\left[\left\|y_{n}(s)\right\|_{\mathcal{L}^{4}}^{4}\right] \mathrm{d} s<\infty
$$

Next we want to study the martingale problem associated to our solutions $\tilde{y}_{n}$ and prove that their limit solves a martingale problem as well, giving existence of a weak solution to the STMHD equations. To this end, take any $\varphi \in C_{c}^{\infty}(\mathbb{R}), e \in \mathcal{E}, t \geq 0, y \in \mathcal{X}$. We define the following process:

$$
M_{e}^{\varphi}(t, y):=I_{1}^{\varphi}(t, y)-I_{2}^{\varphi}(t, y)-I_{3}^{\varphi}(t, y)-I_{4}^{\varphi}(t, y)-I_{5}^{\varphi}(t, y)
$$

where

$$
\begin{aligned}
I_{1}^{\varphi}(t, y) & :=\varphi\left(\langle y(t), e\rangle_{\mathcal{H}^{1}}\right) \\
I_{2}^{\varphi}(t, y) & :=\varphi\left(\langle y(0), e\rangle_{\mathcal{H}^{1}}\right) \\
I_{3}^{\varphi}(t, y): & \int_{0}^{t} \varphi^{\prime}\left(\langle y(0), e\rangle_{\mathcal{H}^{1}}\right) \cdot\langle\mathcal{A}(y(s)), e\rangle_{\mathcal{H}^{1}} \mathrm{~d} s \\
I_{4}^{\varphi}(t, y): & \int_{0}^{t} \varphi^{\prime}\left(\langle y(0), e\rangle_{\mathcal{H}^{1}}\right) \cdot\langle f(s, y(s)), e\rangle_{\mathcal{H}^{1}} \mathrm{~d} s \\
I_{5}^{\varphi}(t, y) & :=\frac{1}{2} \int_{0}^{t} \varphi^{\prime \prime}\left(\langle y(0), e\rangle_{\mathcal{H}^{1}}\right) \cdot\left\|\langle\mathcal{B}(s, y(s)), e\rangle_{\mathcal{H}^{1}}\right\|_{\ell^{2}}^{2} \mathrm{~d} s .
\end{aligned}
$$


Our aim now is to show that $M_{e}^{\varphi}(\cdot, y)$ is a martingale with respect to the stochastic basis $\left(\mathcal{X}, \mathcal{B}(\mathcal{X}), \mu,\left(\mathcal{B}_{t}(\mathcal{X})\right)_{t \geq 0}\right)$, i.e. it solves the martingale problem. This implies the existence of a weak solution.

Since $e \in \mathcal{E} \subset \mathcal{V}$, as noted before, it has compact support, i.e. there exists an $m \in \mathbb{N}$ such that $\operatorname{supp}(e) \subset \mathcal{O}:=\overline{B_{m}(0)} \subset \mathbb{R}^{3}$.

In order to show convergence of the martingale problems, we need to prove uniform moment estimates as well as convergence in probability. The next lemma provides the moment estimates.

Lemma 3.12 (Uniform integrability of $M_{e}^{\varphi}$ ) The following estimate holds

$$
\sup _{n \in \mathbb{N}} \mathbb{E}^{\tilde{P}}\left[\left|M_{e}^{\varphi}\left(t, \tilde{y}_{n}\right)\right|^{4 / 3}\right]+\mathbb{E}^{\tilde{P}}\left[\left|M_{e}^{\varphi}(t, \tilde{y})\right|^{4 / 3}\right]<\infty
$$

Proof We show that each of the terms of $M_{e}^{\varphi}$ are bounded. The terms $I_{1,2}^{\varphi}$ are obviously bounded by a constant $C_{\varphi}$, since $\varphi \in C_{c}^{\infty}$.

For $I_{3}^{\varphi}$ we have by Jensen's inequality for the temporal integral, as well as Eqs. (14) and (6)

$$
\begin{aligned}
\mathbb{E}^{\tilde{P}}\left[\left|I_{3}^{\varphi}\left(t, \tilde{y}_{n}\right)\right|^{4 / 3}\right] & \leq T^{4 / 3-1}\left\|\varphi^{\prime}\right\|_{L^{\infty}}^{4 / 3} \int_{0}^{T} \mathbb{E}^{\tilde{P}}\left[\left|\left\langle\mathcal{A}\left(\tilde{y}_{n}(s)\right), e\right\rangle_{\mathcal{H}^{1}}\right|^{4 / 3}\right] \mathrm{d} s \\
& \leq C_{T, \varphi, e} \int_{0}^{T} \mathbb{E}^{\tilde{P}}\left[1+\left\|\tilde{y}_{n}(s)\right\|_{\mathcal{L}^{3}(\mathcal{O})}^{4}\right] \mathrm{d} s \\
& \leq C_{T, \varphi, e} \int_{0}^{T} \mathbb{E}^{\tilde{P}}\left[1+\left\|\tilde{y}_{n}(s)\right\|_{\mathcal{L}^{12}(\mathcal{O})}^{4}\right] \mathrm{d} s \\
& \leq C_{T, \varphi, e} \int_{0}^{T} \mathbb{E}^{\tilde{P}}\left[1+\|\tilde{\boldsymbol{v}}\| \nabla \tilde{\boldsymbol{v}}\left|\left\|_{L^{2}}^{2}+\right\|\right| \tilde{\boldsymbol{B}}\|\nabla \tilde{\boldsymbol{B}} \mid\|_{L^{2}}^{2}\right] \mathrm{d} s .
\end{aligned}
$$

This last term is bounded by our a priori estimates (32).

In the case of periodic domain $\mathbb{D}=\mathbb{T}^{3}$, we have by Eqs. (14) and (33)

$$
\begin{aligned}
\mathbb{E}^{\tilde{P}}\left[\left|I_{3}^{\varphi}\left(t, \tilde{y}_{n}\right)\right|^{4 / 3}\right] & \leq T^{4 / 3-1}\|\varphi\|_{L^{\infty}}^{4 / 3} \int_{0}^{T} \mathbb{E}^{\tilde{P}}\left[\left|\left\langle\mathcal{A}\left(\tilde{y}_{n}(s)\right), e\right\rangle_{\mathcal{H}^{1}}\right|^{4 / 3}\right] \mathrm{d} s \\
& \leq C_{T, \varphi, e} \int_{0}^{T} \mathbb{E}^{\tilde{P}}\left[1+\left\|\tilde{y}_{n}(s)\right\|_{\mathcal{L}^{3}\left(\mathbb{T}^{3}\right)}^{4}\right] \mathrm{d} s \\
& \leq C_{T, \varphi, e} \int_{0}^{T} \mathbb{E}^{\tilde{P}}\left[1+\left\|\tilde{y}_{n}(s)\right\|_{\mathcal{L}^{4}\left(\mathbb{T}^{3}\right)}^{4}\right] \mathrm{d} s \\
& \leq C_{T, \varphi, e, N, f, H, y_{0}}
\end{aligned}
$$

The other two terms can be dealt with swiftly: by Jensen's inequality for the convex function $x \mapsto x^{4 / 3}$, Hölder's inequality for $p=3 / 2, q=3$, Jensen's inequality for 
the concave function $x \mapsto x^{2 / 3}$, Assumption (H1) and (30) we find

$$
\begin{aligned}
\mathbb{E}^{\tilde{P}}\left[\left|I_{4}^{\varphi}\left(t, \tilde{y}_{n}\right)\right|^{4 / 3}\right] & \leq C_{T, \varphi}\|e\|_{\mathcal{H}^{2}} \int_{0}^{T} \mathbb{E}^{\tilde{P}}\left[1 \cdot\left\|f\left(s, \tilde{y}_{n}(s)\right)\right\|_{\mathcal{H}^{0}}^{4 / 3}\right] \mathrm{d} s \\
& \leq C_{T, \varphi, e} \int_{0}^{T}\left(\mathbb{E}^{\tilde{P}}\left[\left\|f\left(s, \tilde{y}_{n}(s)\right)\right\|_{\mathcal{H}^{0}}^{2}\right]\right)^{2 / 3} \mathrm{~d} s \\
& \leq C_{T, \varphi, e}\left(\int_{0}^{T}\left(1+\mathbb{E}^{\tilde{P}}\left[\left\|\tilde{y}_{n}(s)\right\|_{\mathcal{H}^{0}}^{2}\right]+\left\|F_{f}(s)\right\|_{L^{1}(\mathbb{D})}\right) \mathrm{d} s\right)^{2 / 3} \\
& \leq C_{T, \varphi, e}\left(1+\mathbb{E}^{\tilde{P}}\left[\sup _{s \in[0, T]}\left\|\tilde{y}_{n}(s)\right\|_{\mathcal{H}^{1}}^{2}\right]+\left\|F_{f}\right\|_{L^{1}([0, T] \times \mathbb{D})}\right)^{2 / 3} \\
& \leq C_{T, \varphi, e, N, f, H, y_{0}},
\end{aligned}
$$

and by Eq. (15), we have in a similar manner

$$
\begin{aligned}
\mathbb{E}^{\tilde{P}}\left[\left|I_{5}^{\varphi}\left(t, \tilde{y}_{n}\right)\right|^{4 / 3}\right] & \leq C_{T, \varphi} \int_{0}^{T} \mathbb{E}^{\tilde{P}}\left[\left\|\mathcal{B}\left(s, \tilde{y}_{n}(s)\right)\right\|_{L_{2}\left(\ell^{2} \times \ell^{2} ; \mathcal{H}^{0}\right)}^{2 \cdot 4 / 3}\right] \mathrm{d} s \\
& \leq C_{T, \varphi, e, H, \Sigma} \int_{0}^{T} 1+\mathbb{E}^{\tilde{P}}\left[\left\|\tilde{y}_{n}(s)\right\|_{\mathcal{L}^{2}(\mathcal{O})}^{8 / 3}\right] \mathrm{d} s \\
& \leq C_{T, \varphi, e, H, \Sigma} \int_{0}^{T} 1+\mathbb{E}^{\tilde{P}}\left[\left\|\tilde{y}_{n}(s)\right\|_{\mathcal{L}^{3}(\mathcal{O})}^{4}\right] \mathrm{d} s \\
& \leq C_{T, \varphi, e, H, \Sigma} \int_{0}^{T} 1+\mathbb{E}^{\tilde{P}}\left[\left\|\tilde{y}_{n}(s)\right\|_{\mathcal{L}^{12}(\mathcal{O})}^{4}\right] \mathrm{d} s \\
& \leq C_{T, \varphi, e, H, \Sigma} \int_{0}^{T} \mathbb{E}^{\tilde{P}}\left[1+\left\|\left|\tilde{\boldsymbol{v}}\|\nabla \tilde{\boldsymbol{v}}\|_{L^{2}}^{2}+\left\|\left|\tilde{\boldsymbol{B}}\|\nabla \tilde{\boldsymbol{B}} \mid\|_{L^{2}}^{2}\right] \mathrm{d} s\right.\right.\right.\right. \\
& \leq C_{T, \varphi, e, H, \Sigma, N, y_{0} .}
\end{aligned}
$$

Our next and last ingredient for the existence proof of weak solutions is the convergence in probability of the martingales defined above, as in [39, Lemma 3.12, p. $236 \mathrm{f}$.].

Lemma 3.13 (Convergence in probability) For every $t>0, M_{e}^{\varphi}\left(t, \tilde{y}_{n}\right) \rightarrow M_{e}^{\varphi}(t, \tilde{y})$ in probability, i.e. for every $\varepsilon>0$

$$
\lim _{n \rightarrow \infty} \tilde{P}\left\{\left|M_{e}^{\varphi}\left(t, \tilde{y}_{n}\right)-M_{e}^{\varphi}(t, \tilde{y})\right|>\varepsilon\right\}=0 .
$$

Proof As before, we show convergence of all the terms of $M_{e}^{\varphi}$ and we denote $\mathcal{O}:=$ $\operatorname{supp}(e)$. Note that by (II), and since $\mathcal{O}$ is bounded, we find by the definition of the metric on $\mathcal{X}$ that

$$
\lim _{n \rightarrow \infty} \int_{\mathcal{O}}\left|\tilde{y}_{n}(t, x, \tilde{\omega})-\tilde{y}(t, x, \tilde{\omega})\right|^{2} \mathrm{~d} x=0, \quad \tilde{P}-\text { a.a. } \tilde{\omega} \in \tilde{\Omega} .
$$


This then implies that for $P$-a.a. $\tilde{\omega} \in \tilde{\Omega}$ and all $t \in[0, T]$

$$
\left|\left\langle\tilde{y}_{n}(t, \cdot, \tilde{\omega})-\tilde{y}(t, \cdot, \tilde{\omega}), e\right\rangle_{\mathcal{H}^{1}}\right| \leq\left\|\tilde{y}_{n}(t, \cdot, \tilde{\omega})-\tilde{y}(t, \cdot, \tilde{\omega})\right\|_{\mathcal{L}^{2}(\mathcal{O})}\|e\|_{\mathcal{H}^{2}} \rightarrow 0
$$

as $n \rightarrow \infty$. By the continuity and boundedness of $\varphi$ and Lebesgue's dominated convergence theorem, we find

$$
\lim _{n \rightarrow \infty} \mathbb{E}^{\tilde{P}}\left[\left|I_{1}^{\varphi}\left(t, \tilde{y}_{n}\right)-I_{1}^{\varphi}(t, \tilde{y})\right|\right]=\lim _{n \rightarrow \infty} \mathbb{E}^{\tilde{P}}\left[\left|\varphi\left(\left\langle\tilde{y}_{n}(t), e\right\rangle_{\mathcal{H}^{1}}\right)-\varphi\left(\langle\tilde{y}(t), e\rangle_{\mathcal{H}^{1}}\right)\right|\right]=0
$$

Similarly one can show

$$
\lim _{n \rightarrow \infty} \mathbb{E}^{\tilde{P}}\left[\left|I_{2}^{\varphi}\left(t, \tilde{y}_{n}\right)-I_{2}^{\varphi}(t, \tilde{y})\right|\right]=\lim _{n \rightarrow \infty} \mathbb{E}^{\tilde{P}}\left[\left|\varphi\left(\left\langle\tilde{y}_{n}(0), e\right\rangle_{\mathcal{H}^{1}}\right)-\varphi\left(\langle\tilde{y}(0), e\rangle_{\mathcal{H}^{1}}\right)\right|\right]=0
$$

For $I_{3}^{\varphi}$, we define for any $R>0$ and $n \in \mathbb{N}$ the stopping time

$$
\tilde{\tau}_{R}^{n}:=\inf \left\{t \geq 0 \mid\left\|\tilde{y}_{n}(t)\right\|_{\mathcal{H}^{1}} \geq R\right\}
$$

Then by Chebychev's inequality, (I) and (24), we get

$$
\begin{aligned}
\sup _{n \in \mathbb{N}} \tilde{P}\left\{\tilde{\tau}_{R}^{n} \leq T\right\} & =\sup _{n \in \mathbb{N}} \tilde{P}\left\{\left\|\tilde{y}_{n}(t)\right\|_{\mathcal{H}^{1}} \geq R\right\} \leq \sup _{n \in \mathbb{N}} \frac{1}{R^{2}} \mathbb{E}^{\tilde{P}}\left[\left\|\tilde{y}_{n}(t)\right\|_{\mathcal{H}^{1}}^{2}\right] \\
& \leq \sup _{n \in \mathbb{N}} \frac{1}{R^{2}} \mathbb{E}^{P}\left[\left\|y_{n}(t)\right\|_{\mathcal{H}^{1}}^{2}\right] \leq \frac{C_{T, N, f, H, y_{0}}}{R^{2}} .
\end{aligned}
$$

For arbitrary $R>0$ we thus find

$$
\begin{aligned}
& \lim _{n \rightarrow \infty} \tilde{P}\left\{\left|I_{3}^{\varphi}\left(t, \tilde{y}_{n}\right)-I_{3}^{\varphi}(t, \tilde{y})\right|>\varepsilon\right\} \\
& \quad \leq \lim _{n \rightarrow \infty} \tilde{P}\left\{\left|I_{3}^{\varphi}\left(t, \tilde{y}_{n}\right)-I_{3}^{\varphi}(t, \tilde{y})\right|>\varepsilon, \tilde{\tau}_{R}^{n}>T\right\}+\frac{C_{T, N, f, H, y_{0}}}{R^{2}} .
\end{aligned}
$$

Equations (14), (16) of Lemma 2.5 - and again the continuity and boundedness of $\varphi$-imply that for any $t \in[0, T]$ and all $\tilde{\omega} \in \tilde{\Omega}$ with $\tilde{\tau}_{R}^{n}(\tilde{\omega})>T$

$$
\begin{aligned}
& \left|\varphi^{\prime}\left(\left\langle\tilde{y}_{n}(t, \tilde{\omega}), e\right\rangle_{\mathcal{H}^{1}}\right)\left\langle\mathcal{A}\left(\tilde{y}_{n}(t, \tilde{\omega})\right), e\right\rangle_{\mathcal{H}^{1}}-\varphi^{\prime}\left(\langle\tilde{y}(t, \tilde{\omega}), e\rangle_{\mathcal{H}^{1}}\right)\langle\mathcal{A}(\tilde{y}(t, \tilde{\omega})), e\rangle_{\mathcal{H}^{1}}\right| \\
& \leq\left|\varphi^{\prime}\left(\left\langle\tilde{y}_{n}(t, \tilde{\omega}), e\right\rangle_{\mathcal{H}^{1}}\right)-\varphi^{\prime}\left(\langle\tilde{y}(t, \tilde{\omega}), e\rangle_{\mathcal{H}^{1}}\right)\right|\left|\left\langle\mathcal{A}\left(\tilde{y}_{n}(t, \tilde{\omega})\right), e\right\rangle_{\mathcal{H}^{1}}\right| \\
& \quad+\left|\varphi^{\prime}\left(\langle\tilde{y}(t, \tilde{\omega}), e\rangle_{\mathcal{H}^{1}}\right)\right|\left|\left\langle\mathcal{A}(\tilde{y}(t, \tilde{\omega}))-\mathcal{A}\left(\tilde{y}_{n}(t, \tilde{\omega})\right), e\right\rangle_{\mathcal{H}^{1}}\right| \rightarrow 0, \text { as } n \rightarrow \infty
\end{aligned}
$$


This-combined with Markov's inequality and Lebesgue's dominated convergence theorem-yields the desired convergence:

$$
\begin{aligned}
& \lim _{n \rightarrow \infty} \tilde{P}\left\{\left|I_{3}^{\varphi}\left(t, \tilde{y}_{n}\right)-I_{3}^{\varphi}(t, \tilde{y})\right|>\varepsilon\right\} \\
& \leq \lim _{R \rightarrow \infty} \lim _{n \rightarrow \infty} \tilde{P}\left\{\left|I_{3}^{\varphi}\left(t, \tilde{y}_{n}\right)-I_{3}^{\varphi}(t, \tilde{y})\right|>\varepsilon, \tilde{\tau}_{R}^{n}>T\right\} \\
& \leq \lim _{R \rightarrow \infty} \lim _{n \rightarrow \infty} \frac{1}{\varepsilon} \mathbb{E}^{\tilde{P}}\left[1_{\left\{\tilde{\tau}_{R}^{n}>T\right\}}\left|I_{3}^{\varphi}\left(t, \tilde{y}_{n}\right)-I_{3}^{\varphi}(t, \tilde{y})\right|\right] \\
& \leq \lim _{R \rightarrow \infty} \frac{1}{\varepsilon} \mathbb{E}^{\tilde{P}}\left[\lim _{n \rightarrow \infty} \int_{0}^{t} 1_{\left\{\tilde{\tau}_{R}^{n}>T\right\}} \cdot \mid \varphi^{\prime}\left(\left\langle\tilde{y}_{n}(t), e\right\rangle_{\mathcal{H}^{1}}\right)\left\langle\mathcal{A}\left(\tilde{y}_{n}(t)\right), e\right\rangle_{\mathcal{H}^{1}}\right. \\
& \left.\quad-\varphi^{\prime}\left(\langle\tilde{y}(t), e\rangle_{\mathcal{H}^{1}}\right)\langle\mathcal{A}(\tilde{y}(t)), e\rangle_{\mathcal{H}^{1}} \mid \mathrm{d} s\right]=0 .
\end{aligned}
$$

Similarly, for $I_{4}^{\varphi}$ we get by Assumption (H1) and similar convergence arguments that

$$
\lim _{n \rightarrow \infty} \tilde{P}\left\{\left|I_{4}^{\varphi}\left(t, \tilde{y}_{n}\right)-I_{4}^{\varphi}(t, \tilde{y})\right|>\varepsilon\right\}=0 .
$$

Finally, for $I_{5}^{\varphi}$ we find that since

$$
\begin{aligned}
&\left|\left\|\left\langle\mathcal{B}\left(s, \tilde{y}_{n}(s)\right), e\right\rangle_{\mathcal{H}^{1}}\right\|_{\ell^{2}}^{2}-\left\|\langle\mathcal{B}(s, \tilde{y}(s)), e\rangle_{\mathcal{H}^{1}}\right\|_{\ell^{2}}^{2}\right| \\
& \leq\left.\left|\sum_{k=1}^{\infty}\right|\left\langle\mathcal{B}_{k}\left(s, \tilde{y}_{n}(s)\right), e\right\rangle_{\mathcal{H}^{1}}\right|^{2}-\left|\left\langle\mathcal{B}_{k}(s, \tilde{y}(s)), e\right\rangle_{\mathcal{H}^{1}}\right|^{2} \mid \\
& \leq\left|\sum_{k=1}^{\infty}\right|\left\langle\mathcal{B}_{k}\left(s, \tilde{y}_{n}(s)\right)-\mathcal{B}_{k}(s, \tilde{y}(s)), e\right\rangle_{\mathcal{H}^{1}}|\cdot|\left\langle\mathcal{B}_{k}\left(s, \tilde{y}_{n}(s)\right), e\right\rangle_{\mathcal{H}^{1}} \\
& \quad+\left\langle\mathcal{B}_{k}(s, \tilde{y}(s)), e\right\rangle_{\mathcal{H}^{1}} \| \\
& \leq\|e\|_{\mathcal{H}^{2}}\left|\sum_{k=1}^{\infty}\right|\left\langle\mathcal{B}_{k}\left(s, \tilde{y}_{n}(s)\right)-\mathcal{B}_{k}(s, \tilde{y}(s)), e\right\rangle_{\mathcal{H}^{1}} \mid \cdot\left(\left\|\mathcal{B}_{k}\left(s, \tilde{y}_{n}(s)\right)\right\|_{\mathcal{H}^{0}}\right. \\
&\left.\quad+\left\|\mathcal{B}_{k}(s, \tilde{y}(s))\right\|_{\mathcal{H}^{0}}\right) \mid \\
& \leq\left.C_{e} \|\left\langle\mathcal{B}\left(s, \tilde{y}_{n}(s)\right)-\mathcal{B}_{(s, \tilde{y}}(s)\right), e\right\rangle_{\mathcal{H}^{1}} \|_{\ell^{2}} \cdot\left(\left\|\mathcal{B}\left(s, \tilde{y}_{n}(s)\right)\right\|_{L_{2}\left(\ell^{2} \times \ell^{2} ; \mathcal{H}^{0}\right)}\right. \\
&\left.+\|\mathcal{B}(s, \tilde{y}(s))\|_{L_{2}\left(\ell^{2} \times \ell^{2} ; \mathcal{H}^{0}\right)}\right)
\end{aligned}
$$

when combining this with (17), the convergence

$$
\lim _{n \rightarrow \infty} \tilde{P}\left\{\left|I_{5}^{\varphi}\left(t, \tilde{y}_{n}\right)-I_{5}^{\varphi}(t, \tilde{y})\right|>\varepsilon\right\}=0
$$

follows. This concludes the proof.

We are now in a position to prove the main result of this section. 
Proof of Theorem 3.9 Let $t>s, G$ be a bounded $\mathbb{R}$-valued, $\mathcal{B}_{s}(\mathcal{X})$-measurable continuous function on $\mathcal{X}$. Then we have by the generalised Lebesgue theorem

$$
\begin{aligned}
& \mathbb{E}^{\mu}\left[\left(M_{e}^{\varphi}(t, y)-M_{e}^{\varphi}(s, y)\right) \cdot G(y)\right] \\
& =\mathbb{E}^{\tilde{P}}\left[\left(M_{e}^{\varphi}(t, \tilde{y})-M_{e}^{\varphi}(s, \tilde{y})\right) \cdot G(\tilde{y})\right] \quad\left(\mu=\tilde{P} \circ \tilde{y}^{-1}\right) \\
& =\lim _{n \rightarrow \infty} \mathbb{E}^{\tilde{P}}\left[\left(M_{e}^{\varphi}\left(t, \tilde{y}_{n}\right)-M_{e}^{\varphi}\left(s, \tilde{y}_{n}\right)\right) \cdot G\left(\tilde{y}_{n}\right)\right] \\
& =\lim _{n \rightarrow \infty} \mathbb{E}^{P}\left[\left(M_{e}^{\varphi}\left(t, y_{n}\right)-M_{e}^{\varphi}\left(s, y_{n}\right)\right) \cdot G\left(y_{n}\right)\right] \quad\left(\tilde{P} \circ \tilde{y}_{n}^{-1}=P \circ y_{n}^{-1}\right) \\
& =0 \text {. }
\end{aligned}
$$

The last step uses the martingale property of $M_{e}^{\varphi}\left(s, y_{n}\right)$ on the stochastic basis $\left(\Omega, \mathcal{F}, P,\left(\mathcal{F}_{t}\right)_{t \geq 0}\right)$ and since $G\left(y_{n}\right)$ is $\mathcal{F}_{s}$-measurable. Therefore, $\mu$ solves the martingale problem and by Proposition 3.3 we infer the existence of a weak solution to (9).

\subsection{Proof of Theorem 1.1}

Proof of Theorem 1.1 The only thing left to prove is estimate (3). By Itô's formula, taking expectations and using (11), Assumption (H1) and Equation (17), we find

$$
\begin{aligned}
\mathbb{E}\|y(t)\|_{\mathcal{H}^{0}}^{2}= & \left\|y_{0}\right\|_{\mathcal{H}^{0}}^{2}+2 \int_{0}^{t} \mathbb{E}\langle\mathcal{A}(y(s)), y(s)\rangle_{\mathcal{H}^{0}} \mathrm{~d} s \\
& +2 \int_{0}^{t} \mathbb{E}\langle f(s, y(s)), y(s)\rangle_{\mathcal{H}^{0}} \mathrm{~d} s+\int_{0}^{t} \mathbb{E}\|\mathcal{B}(s, y(s))\|_{L_{2}\left(\ell^{2} \times \ell^{2} ; \mathcal{H}^{0}\right)}^{2} \mathrm{~d} s \\
& \leq\left\|y_{0}\right\|_{\mathcal{H}^{0}}^{2}-\frac{3}{2} \int_{0}^{t} \mathbb{E}\|y(s)\|_{\mathcal{H}^{1}}^{2} \mathrm{~d} s+C_{f, H}+C_{T, f, H} \int_{0}^{t} \mathbb{E}\|y(s)\|_{\mathcal{H}^{0}}^{2} \mathrm{~d} s
\end{aligned}
$$

which by Gronwall's lemma implies

$$
\mathbb{E}\|y(t)\|_{\mathcal{H}^{0}}^{2}+\int_{0}^{t} \mathbb{E}\|y(s)\|_{\mathcal{H}^{1}}^{2} \mathrm{~d} s \leq C_{T, f, H}\left(\left\|y_{0}\right\|_{\mathcal{H}^{0}}^{2}+1\right)
$$

Applying Burkholder's inequality and performing similar calculations as in the proof of Lemma 3.10, we get the desired estimate.

\section{Feller property and existence of invariant measures}

In this section we prove further properties of the stochastic tamed MHD equations. First, we show that they generate a Feller semigroup under stronger assumptions. 
Then, we prove that there exists an invariant measure for this Feller semigroup in the case of periodic boundary conditions.

We consider the time-homogeneous case, i.e. the functions $f, \Sigma, H$ of our equations are assumed to be independent of time. Furthermore, we assume Lipschitz conditions on the first-order derivatives of the function $H$ :

(H3)' There exists a constant $C_{H}>0$ and a function $F_{H} \in L^{1}(\mathbb{D})$ such that for all $x \in \mathbb{D}, y, y^{\prime} \in \mathbb{R}^{6}, j=1,2,3$ the following conditions hold:

$$
\begin{aligned}
\left\|\partial_{x^{j}} H(x, y)\right\|_{\ell^{2}}^{2}+\|H(x, y)\|_{\ell^{2}}^{2} & \leq C_{H}|y|^{2}+F_{H}(x), \\
\left\|\partial_{x^{j}} H(x, y)-\partial_{x^{j}} H\left(x, y^{\prime}\right)\right\|_{\ell^{2}} & \leq C_{H}\left|y-y^{\prime}\right|, \\
\left\|\partial_{y^{j}} H(x, y)\right\|_{\ell^{2}} & \leq C_{H}, \\
\left\|\partial_{y^{j}} H(x, y)-\partial_{\left(y^{\prime}\right)^{j}} H\left(x, y^{\prime}\right)\right\|_{\ell^{2}} & \leq C_{H}\left|y-y^{\prime}\right| .
\end{aligned}
$$

For an initial condition $y_{0} \in \mathcal{H}^{1}$, let $y\left(t ; y_{0}\right)$ be the unique solution to (9) with $y\left(0 ; y_{0}\right)=y_{0}$. Then by the uniqueness of solutions, we know that $\left\{y\left(t ; y_{0}\right) \mid y_{0} \in\right.$ $\left.\mathcal{H}^{1}, t \geq 0\right\}$ is a strong Markov process with state space $\mathcal{H}^{1}$. In proving the Feller property of the associated semigroup, we need the following result.

Lemma 4.1 For $y_{0}, y_{0}^{\prime} \in \mathcal{H}^{1}, R>0$, define the stopping times

$$
\begin{aligned}
\tau_{R}^{y_{0}} & :=\inf \left\{t \geq 0 \mid\left\|y\left(t ; y_{0}\right)\right\|_{\mathcal{H}^{1}}>R\right\}, \\
\tau_{R}:=\tau_{R}^{y_{0}, y_{0}^{\prime}} & :=\tau_{R}^{y_{0}} \wedge \tau_{R}^{y_{0}^{\prime}} .
\end{aligned}
$$

Assume (H1), (H2), (H3)'. Then there is a constant $C_{t, R, N, f, H, \Sigma}>0$ such that

$$
\mathbb{E}\left[\left\|y\left(t \wedge \tau_{R} ; y_{0}\right)-y\left(t \wedge \tau_{R} ; y_{0}^{\prime}\right)\right\|_{\mathcal{H}^{1}}^{2}\right] \leq C_{t, R, N, f, H, \Sigma}\left\|y_{0}-y_{0}^{\prime}\right\|_{\mathcal{H}^{1}}^{2} .
$$

Proof We follow the proof of [39, Lemma 4.1, p. $238 \mathrm{ff}$.] For notational convenience, we denote $y(t):=y\left(t ; y_{0}\right), \tilde{y}(t):=y\left(t ; y_{0}^{\prime}\right), z(t):=y(t)-\tilde{y}(t)$ and $t_{R}:=t \wedge \tau_{R}$. By Itô's formula, we have

$$
\begin{aligned}
\left\|z\left(t_{R}\right)\right\|_{\mathcal{H}^{1}}^{2}= & \|z(0)\|_{\mathcal{H}^{1}}^{2}+2 \int_{0}^{t_{R}}\langle\mathcal{A}(y(s))-\mathcal{A}(\tilde{y}(s)), z(s)\rangle_{\mathcal{H}^{1}} \mathrm{~d} s \\
& +2 \int_{0}^{t_{R}}\langle f(y(s))-f(\tilde{y}(s)), z(s)\rangle_{\mathcal{H}^{1}} \mathrm{~d} s \\
& +2 \sum_{k=1}^{\infty} \int_{0}^{t_{R}}\left\langle\mathcal{B}_{k}(y(s))-\mathcal{B}_{k}(\tilde{y}(s)), z(s)\right\rangle_{\mathcal{H}^{1}} \mathrm{~d} \mathcal{W}_{s}^{k}
\end{aligned}
$$




$$
\begin{aligned}
& +\sum_{k=1}^{\infty} \int_{0}^{t_{R}}\left\|\mathcal{B}_{k}(y(s))-\mathcal{B}_{k}(\tilde{y}(s)), z(s)\right\|_{\mathcal{H}^{1}}^{2} \mathrm{~d} s \\
= & :\|z(0)\|_{\mathcal{H}^{1}}^{2}+I_{1}\left(t_{R}\right)+I_{2}\left(t_{R}\right)+I_{3}\left(t_{R}\right)+I_{4}\left(t_{R}\right) .
\end{aligned}
$$

We denote by $\boldsymbol{z}_{v}$ the velocity component of $z$, i.e. $\boldsymbol{z}_{v}:=\boldsymbol{v}-\tilde{\boldsymbol{v}}$, and similarly we write $z_{B}:=\boldsymbol{B}-\tilde{\boldsymbol{B}}$. Then $I_{1}\left(t_{R}\right)$ has the following form:

$$
\begin{aligned}
I_{1}\left(t_{R}\right)= & 2 \int_{0}^{t_{R}}\langle\Delta z, z\rangle_{\mathcal{H}^{1}}-2 \int_{0}^{t_{R}}\left\langle g_{N}\left(|y|^{2}\right) y-g_{N}\left(|\tilde{y}|^{2}\right) \tilde{y}, z\right\rangle_{\mathcal{H}^{1}} \\
& +2 \int_{0}^{t_{R}}\left\{\left\langle-(\boldsymbol{v} \cdot \nabla) \boldsymbol{v}+(\tilde{\boldsymbol{v}} \cdot \nabla) \tilde{\boldsymbol{v}}, z_{v}\right\rangle_{\mathcal{H}^{1}}+\left\langle(\boldsymbol{B} \cdot \nabla) \boldsymbol{B}-(\tilde{\boldsymbol{B}} \cdot \nabla) \tilde{\boldsymbol{B}}, \boldsymbol{z}_{v}\right\rangle_{\mathcal{H}^{1}}\right. \\
& \left.+\left\langle-(\boldsymbol{v} \cdot \nabla) \boldsymbol{B}+(\tilde{\boldsymbol{v}} \cdot \nabla) \tilde{\boldsymbol{B}}, \boldsymbol{z}_{B}\right\rangle_{\mathcal{H}^{1}}+\left\langle(\boldsymbol{B} \cdot \nabla) \boldsymbol{v}-(\tilde{\boldsymbol{B}} \cdot \nabla) \tilde{\boldsymbol{v}}, \boldsymbol{z}_{B}\right\rangle_{\mathcal{H}^{1}}\right\} \mathrm{d} s .
\end{aligned}
$$

The first term is readily analysed:

$$
2 \int_{0}^{t_{R}}\langle\Delta z, z\rangle_{\mathcal{H}^{1}}=-2 \int_{0}^{t_{R}}\|z\|_{\mathcal{H}^{2}}^{2} \mathrm{~d} s+2 \int_{0}^{t_{R}}\|z\|_{\mathcal{H}^{1}}^{2} \mathrm{~d} s
$$

For the second term, we find by using Young's inequality, $g_{N}(r) \leq \mathrm{Cr}$, Hölder's inequality (with $p=3, q=3 / 2$ ) and the Sobolev embedding $\mathcal{H}^{1} \subset \overline{\mathcal{L}^{6}}$ that for some $\theta \in \mathbb{R}$

$$
\begin{aligned}
& \left\langle g_{N}\left(|y|^{2}\right) y-g_{N}\left(|\tilde{y}|^{2}\right) \tilde{y}, z\right\rangle_{\mathcal{H}^{1}} \\
& \quad \leq\left\|g_{N}\left(|y|^{2}\right)|z|\right\|_{L^{2}}\|z\|_{\mathcal{H}^{2}}+\left\|\left|g_{N}^{\prime}(\theta)\right| \cdot|z|(|y|+|\tilde{y}|)|\tilde{y}|\right\|_{L^{2}}\|z\|_{\mathcal{H}^{2}} \\
& \quad \leq 2 \varepsilon\|z\|_{\mathcal{H}^{2}}^{2}+C_{\varepsilon, N}\left(\left\|g_{N}\left(|y|^{2}\right)|z|\right\|_{L^{2}}^{2}+\left\||z|\left(|y|^{2}+|\tilde{y}|^{2}\right)\right\|_{L^{2}}^{2}\right) \\
& \quad \leq 2 \varepsilon\|z\|_{\mathcal{H}^{2}}^{2}+C_{\varepsilon, N}\|z\|_{\mathcal{L}^{6}}^{2}\left(\|y\|_{\mathcal{L}^{6}}^{4}+\|\tilde{y}\|_{\mathcal{L}^{6}}^{4}\right) \\
& \quad \leq 2 \varepsilon\|z\|_{\mathcal{H}^{2}}^{2}+C_{\varepsilon, N}\|z\|_{\mathcal{H}^{1}}^{2}\left(\|y\|_{\mathcal{H}^{1}}^{4}+\|\tilde{y}\|_{\mathcal{H}^{1}}^{4}\right) \\
& \quad \leq 2 \varepsilon\|z\|_{\mathcal{H}^{2}}^{2}+C_{\varepsilon, N, R}\|z\|_{\mathcal{H}^{1}}^{2} .
\end{aligned}
$$

The third term consists of four sub-terms, all of which are very similar. We thus only estimate one of them: Young's inequality, the Sobolev embedding $H^{1} \subset L^{6}$ as well as the Gagliardo-Nirenberg-Sobolev inequalities $\|\boldsymbol{u}\|_{L^{\infty}} \leq C\|\boldsymbol{u}\|_{H^{2}}^{3 / 4}\|\boldsymbol{u}\|_{L^{2}}^{1 / 4}$ and $\|\nabla \boldsymbol{u}\|_{L^{3}} \leq C\|\boldsymbol{u}\|_{H^{2}}^{3 / 4}\|\boldsymbol{u}\|_{L^{2}}^{1 / 4}$, combined with another application of Young's inequality 
(with $p=4 / 3, q=4$ ) yield

$$
\begin{aligned}
& \left\langle(\boldsymbol{v} \cdot \nabla) \boldsymbol{v}-(\tilde{\boldsymbol{v}} \cdot \nabla) \tilde{\boldsymbol{v}}, \boldsymbol{z}_{v}\right\rangle_{H^{1}} \leq 2 \varepsilon\left\|\boldsymbol{z}_{v}\right\|_{H^{2}}^{2}+C_{\varepsilon}\left(\left\|\left(\boldsymbol{z}_{v} \cdot \nabla\right) \boldsymbol{v}\right\|_{L^{2}}^{2}+\left\|(\tilde{\boldsymbol{v}} \cdot \nabla) \boldsymbol{z}_{v}\right\|_{L^{2}}^{2}\right) \\
& \quad \leq 2 \varepsilon\left\|\boldsymbol{z}_{v}\right\|_{H^{2}}^{2}+C_{\varepsilon}\left(\left\|\boldsymbol{z}_{v}\right\|_{L^{\infty}}^{2}\|\nabla \boldsymbol{v}\|_{L^{2}}^{2}+\|\tilde{\boldsymbol{v}}\|_{L^{6}}^{2}\left\|\nabla \boldsymbol{z}_{v}\right\|_{L^{3}}^{2}\right) \\
& \quad \leq 2 \varepsilon\left\|\boldsymbol{z}_{v}\right\|_{H^{2}}^{2}+C_{\varepsilon}\left(\left\|\boldsymbol{z}_{v}\right\|_{H^{2}}^{3 / 2}\left\|\boldsymbol{z}_{v}\right\|_{L^{2}}^{1 / 2}\|\boldsymbol{v}\|_{H^{1}}^{2}+\|\tilde{\boldsymbol{v}}\|_{H^{1}}^{2}\left\|z_{v}\right\|_{H^{2}}^{3 / 2}\left\|\boldsymbol{z}_{v}\right\|_{L^{2}}^{1 / 2}\right) \\
& \quad \leq 4 \varepsilon\left\|\boldsymbol{z}_{v}\right\|_{H^{2}}^{2}+C_{\varepsilon, R}\left\|\boldsymbol{z}_{v}\right\|_{L^{2}}^{2} \leq 4 \varepsilon\left\|\boldsymbol{z}_{v}\right\|_{H^{2}}^{2}+C_{\varepsilon, R}\left\|\boldsymbol{z}_{v}\right\|_{H^{1}}^{2} .
\end{aligned}
$$

Analysing the other sub-terms in the same way and putting everything together, we find

$$
I_{1}\left(t_{R}\right) \leq-2 \int_{0}^{t_{R}}\|z(s)\|_{\mathcal{H}^{2}}^{2} \mathrm{~d} s+C_{\varepsilon, N, R} \int_{0}^{t_{R}}\|z(s)\|_{\mathcal{H}^{1}}^{2} \mathrm{~d} s+10 \varepsilon \int_{0}^{t_{R}}\|z(s)\|_{\mathcal{H}^{2}}^{2} \mathrm{~d} s .
$$

$I_{2}\left(t_{R}\right)$ is estimated using Young's inequality and Assumption (H1)

$$
\begin{aligned}
I_{2}\left(t_{R}\right) & \leq \varepsilon \int_{0}^{t_{R}}\|z(s)\|_{\mathcal{H}^{2}}^{2} \mathrm{~d} s+C_{\varepsilon} \int_{0}^{t_{R}}\|f(y(s))-f(\tilde{y}(s))\|_{\mathcal{H}^{0}}^{2} \mathrm{~d} s \\
& \leq \varepsilon \int_{0}^{t_{R}}\|z(s)\|_{\mathcal{H}^{2}}^{2} \mathrm{~d} s+C_{\varepsilon, f} \int_{0}^{t_{R}}\|z(s)\|_{\mathcal{H}^{0}}^{2} \mathrm{~d} s .
\end{aligned}
$$

The term $I_{3}\left(t_{R}\right)$ is a martingale and thus vanishes after taking expectations.

For $I_{4}\left(t_{R}\right)$, we use the following considerations, similar to the ones in the proof of Lemma 2.6:

$$
\begin{aligned}
& \|\mathcal{B}(y)-\mathcal{B}(\tilde{y})\|_{L_{2}\left(\ell^{2} \times \ell^{2} ; \mathcal{H}^{1}\right)}^{2} \\
& \quad=\|\mathcal{B}(y)-\mathcal{B}(\tilde{y})\|_{L_{2}\left(\ell^{2} \times \ell^{2} ; \mathcal{H}^{0}\right)}^{2}+\|\nabla(\mathcal{B}(y)-\mathcal{B}(\tilde{y}))\|_{L_{2}\left(\ell^{2} \times \ell^{2} ; \mathcal{H}^{0}\right)}^{2}
\end{aligned}
$$

and the latter term consists (by using the chain rule) of terms of the following form:

$$
\begin{aligned}
\partial_{x^{j}} & \left(\mathcal{B}_{k}(y)-\mathcal{B}_{k}(\tilde{y})\right)=\partial_{x^{j}}\left(\mathcal{P}\left(\Sigma_{k}(x) \cdot \nabla\right) z+\mathcal{P}\left(H_{k}(x, y)-H_{k}(x, \tilde{y})\right)\right) \\
= & \mathcal{P}\left\{\left(\left(\partial_{x^{j}} \Sigma_{k}(x)\right) \cdot \nabla\right) z+\left(\Sigma_{k}(x) \cdot \nabla\right) \partial_{x^{j}} z+\left(\partial_{x^{j}} H_{k}\right)(x, y)-\left(\partial_{x^{j}} H_{k}\right)(x, \tilde{y})\right. \\
& \left.+\sum_{i=1}^{6}\left[\left(\partial_{y^{i}} H_{k}(x, y)\right) \partial_{x^{j}} z^{i}-\left\{\left(\partial_{y^{i}} H_{k}(x, y)\right)-\left(\partial_{\tilde{y}^{i}} H_{k}(x, \tilde{y})\right)\right\} \partial_{x^{j}} \tilde{y}^{i}\right]\right\} .
\end{aligned}
$$


Thus we find, using Assumptions (H2) and (H3)', as well as Eq. (5), the GagliardoNirenberg inequality and Young's inequality, that

$$
\begin{aligned}
& \|\mathcal{B}(y)-\mathcal{B}(\tilde{y})\|_{L_{2}\left(\ell^{2} \times \ell^{2} ; \mathcal{H}^{1}\right)}^{2} \\
& \leq 2 \sum_{k=1}^{\infty}\left\|\left(\Sigma_{k} \cdot \nabla\right) z\right\|_{\mathcal{H}^{0}}^{2}+\left\|H_{k}(y)-H_{k}(\tilde{y})\right\|_{\mathcal{H}^{0}}^{2} \\
& +\sum_{k=1}^{\infty} \int_{\mathbb{D}} \sum_{j=1}^{3} \mid\left(\left(\partial_{x^{j}} \Sigma_{k}(x)\right) \cdot \nabla\right) z+\left(\Sigma_{k}(x) \cdot \nabla\right) \partial_{x^{j}} z \\
& +\left(\partial_{x^{j}} H_{k}\right)(x, y)-\left(\partial_{x^{j}} H_{k}\right)(x, \tilde{y}) \\
& +\left.\sum_{i=1}^{6}\left[\left(\partial_{y^{i}} H_{k}(x, y)\right) \partial_{x^{j}} z^{i}-\left\{\left(\partial_{y^{i}} H_{k}(x, y)\right)-\left(\partial_{\tilde{y}^{i}} H_{k}(x, \tilde{y})\right)\right\} \partial_{x^{j}} \tilde{y}^{i}\right]\right|^{2} \mathrm{~d} x \\
& \leq 2 \sup _{x \in \mathbb{D}}\left\|\Sigma_{k}(x)\right\|_{\ell^{2}}^{2}\|\nabla z\|_{\mathcal{H}^{0}}^{2}+C_{H}\|z\|_{\mathcal{H}^{0}}^{2}+2 \sum_{k=1}^{\infty} \int_{\mathbb{D}} \sum_{j=1}^{3}\left|\left(\Sigma_{k}(x) \cdot \nabla\right) \partial_{x^{j}} z\right|^{2} \mathrm{~d} x \\
& +2 \sum_{k=1}^{\infty} \int_{\mathbb{D}} \sum_{j=1}^{3} \mid\left(\left(\partial_{x^{j}} \Sigma_{k}(x)\right) \cdot \nabla\right) z+\left(\partial_{x^{j}} H_{k}\right)(x, y)-\left(\partial_{x^{j}} H_{k}\right)(x, \tilde{y}) \\
& +\sum_{i=1}^{6}\left(\partial_{y^{i}} H_{k}(x, y)\right) \partial_{x^{j}} z^{i}-\left.\sum_{i=1}^{6}\left\{\left(\partial_{y^{i}} H_{k}(x, y)\right)-\left(\partial_{\tilde{y}^{i}} H_{k}(x, \tilde{y})\right)\right\} \partial_{x^{j}} \tilde{y}^{i}\right|^{2} \mathrm{~d} x \\
& \leq 2 \sup _{x \in \mathbb{D}}\left\|\Sigma_{k}(x)\right\|_{\ell^{2}}^{2}\|z\|_{\mathcal{H}^{1}}^{2}+C_{H}\|z\|_{\mathcal{H}^{0}}^{2}+2 \sup _{x \in \mathbb{D}}\left\|\Sigma_{k}(x)\right\|_{\ell^{2}}^{2} \sum_{j, l=1}^{3}\left\|\partial_{x^{l}} \partial_{x^{j}} z\right\|_{\mathcal{L}^{2}}^{2} \\
& +8 \sup _{x \in \mathbb{D}}\left\|\nabla \Sigma_{k}(x)\right\|_{\ell^{2}}^{2}\|\nabla z\|_{\mathcal{H}^{0}}^{2}+C_{H}\|z\|_{\mathcal{H}^{1}}^{2}+C_{H} \int_{\mathbb{D}} \sum_{j=1}^{3} \sum_{i=1}^{6}|z|^{2}\left|\partial_{x^{j}} \tilde{y}^{i}\right|^{2} \mathrm{~d} x \\
& \leq 2 d^{2} \sup _{x \in \mathbb{D}}\left\|\Sigma_{k}(x)\right\|_{\ell^{2}}^{2}\|z\|_{\mathcal{H}^{2}}^{2}+C_{\Sigma, H}\|z\|_{\mathcal{H}^{1}}^{2}+C_{H}\|z\|_{\mathcal{L}^{\infty}}^{2}\|\tilde{y}\|_{\mathcal{H}^{1}}^{2} \\
& \leq\left(\frac{1}{2}+\varepsilon\right)\|z\|_{\mathcal{H}^{2}}^{2}+C_{\Sigma, H}\|z\|_{\mathcal{H}^{1}}^{2}+C_{H}\|z\|_{\mathcal{H}^{0}}^{2}\|\tilde{y}\|_{\mathcal{H}^{1}}^{8} .
\end{aligned}
$$

Integrating over time we finally get

$$
I_{4}\left(t_{R}\right) \leq \int_{0}^{t_{R}}\left(\frac{1}{2}+\varepsilon\right)\|z\|_{\mathcal{H}^{2}}^{2}+C_{H, R}\|z\|_{\mathcal{H}^{0}}^{2}+C_{\Sigma, H}\|z\|_{\mathcal{H}^{1}}^{2} \mathrm{~d} s .
$$


Thus, adding all the contributions together,

$$
\begin{aligned}
& \mathbb{E}\left[\left\|z\left(t_{R}\right)\right\|_{\mathcal{H}^{1}}^{2}\right] \\
& \quad \leq\|z(0)\|_{\mathcal{H}^{1}}^{2}-(3 / 2-12 \varepsilon) \mathbb{E}\left[\int_{0}^{t_{R}}\|z(s)\|_{\mathcal{H}^{2}}^{2} \mathrm{~d} s\right]+C_{\varepsilon, R, N, f, H, \Sigma} \mathbb{E}\left[\int_{0}^{t_{R}}\|z(s)\|_{\mathcal{H}^{1}}^{2} \mathrm{~d} s\right] .
\end{aligned}
$$

Choosing $\varepsilon=\frac{1}{8}$, we find

$$
\mathbb{E}\left[\left\|z\left(t \wedge \tau_{R}\right)\right\|_{\mathcal{H}^{1}}^{2}\right] \leq\|z(0)\|_{\mathcal{H}^{1}}^{2}+C_{R, N, f, H, \Sigma} \int_{0}^{t} \mathbb{E}\left[\left\|z\left(s \wedge \tau_{R}\right)\right\|_{\mathcal{H}^{1}}^{2} \mathrm{~d} s\right]
$$

An application of Gronwall's Lemma then yields the desired result.

Let $B C_{\text {loc }}\left(\mathcal{H}^{1}\right)$ denote the set of bounded, locally uniformly continuous functions on $\mathcal{H}^{1}$. The supremum norm

$$
\|\phi\|_{\infty}:=\sup _{y \in \mathcal{H}^{1}}|\phi(y)|
$$

turns this space into a Banach space.

For $t \geq 0$, define the semigroup $T_{t}$ associated with the Markov process $\left\{y\left(t ; y_{0}\right) \mid y_{0} \in \mathcal{H}^{1}, t \geq 0\right\}$ by

$$
T_{t} \phi\left(y_{0}\right):=\mathbb{E}\left[\phi\left(y\left(t ; y_{0}\right)\right)\right], \quad \phi \in B C_{\mathrm{loc}}\left(\mathcal{H}^{1}\right) .
$$

Using the previous lemma, we show that this is a Feller semigroup.

Theorem 4.2 (Feller property) Under the Assumptions (H1), (H2) and (H3)', for every $t \geq 0, T_{t}$ maps $B C_{\text {loc }}\left(\mathcal{H}^{1}\right)$ into itself, i.e. it is a Feller semigroup on $B C_{\text {loc }}\left(\mathcal{H}^{1}\right)$.

Proof Having formulated the problem in this abstract way, we can use Lemma 4.1 to run the exact same proof as in [39, Theorem 4.2, p. $241 \mathrm{f}$.] to prove the claim, as it does not use any specific properties of the Navier-Stokes equations.

In the periodic case, we can show existence of an invariant measure for our equations:

Theorem 4.3 (Invariant measures in the periodic case) Under the hypotheses ( $\mathrm{H1}$ ), (H2), (H3)', in the periodic case $\mathbb{D}=\mathbb{T}^{3}$, there exists an invariant measure $\mu \in \mathcal{P}\left(\mathcal{H}^{1}\right)$ associated to $\left(T_{t}\right)_{t \geq 0}$ such that for every $t \geq 0, \phi \in B C_{\text {loc }}\left(\mathcal{H}^{1}\right)$

$$
\int_{\mathcal{H}^{1}} T_{t} \phi\left(y_{0}\right) \mathrm{d} \mu\left(y_{0}\right)=\int_{\mathcal{H}^{1}} \phi\left(y_{0}\right) \mathrm{d} \mu\left(y_{0}\right) .
$$


Proof We start by using Itô's formula for $\|y(t)\|_{\mathcal{H}^{0}}^{2}$, Eq. (12), Young's inequality, Eq. (17) as well as Assumption (H1) and-owing to the boundedness of the domain $\mathbb{T}^{3}$ - the embedding $\mathcal{L}^{4}\left(\mathbb{T}^{3}\right) \subset \mathcal{L}^{2}\left(\mathbb{T}^{3}\right)$. These combined yield that

$$
\mathbb{E}\left[\|y(t)\|_{\mathcal{H}^{0}}^{2}\right]+\int_{0}^{t} \mathbb{E}\left[\|y(s)\|_{\mathcal{H}^{1}}^{2}\right] \mathrm{d} s+\int_{0}^{t} \mathbb{E}\left[\|y(s)\|_{\mathcal{L}^{4}}^{4}\right] \mathrm{d} s \leq C_{f, H, N, \mathbb{D}}\left(\|y(0)\|_{\mathcal{H}^{0}}^{2}+t\right) .
$$

Now using the Itô formula for the norm $\|y(t)\|_{\mathcal{H}^{1}}^{2}$ and the corresponding higher-order estimates (13), (18), we find by proceeding in the same way as before-additionally using (39) - that

$$
\mathbb{E}\left[\|y(t)\|_{\mathcal{H}^{1}}^{2}\right] \leq-\frac{1}{2} \int_{0}^{t} \mathbb{E}\left[\|y(s)\|_{\mathcal{H}^{2}}^{2}\right] \mathrm{d} s+C_{f, H, N, \mathbb{D}} \cdot t
$$

which implies

$$
\frac{1}{t} \int_{0}^{t} \mathbb{E}\left[\|y(s)\|_{\mathcal{H}^{2}}^{2}\right] \mathrm{d} s \leq C_{f, H, N, \mathbb{D} .}
$$

Note that this inequality is uniform in $t$ and in the initial condition $y_{0}$.

We now conclude as follows: following the notation of Da Prato and Zabczyk [11], we define the kernels

$$
R_{T}\left(y_{0}, \Gamma\right):=\frac{1}{T} \int_{0}^{T}\left(T_{S} 1_{\Gamma}\right)\left(y_{0}\right) \mathrm{d} t, \quad y_{0} \in \mathcal{H}^{1}, \Gamma \in \mathcal{B}\left(\mathcal{H}^{1}\right)
$$

Set $E=\mathcal{H}^{1}$ and let $v \in \mathcal{P}\left(\mathcal{H}^{1}\right)$ be an arbitrary probability measure. Define the associated measure

$$
R_{T}^{*} v(\Gamma)=\int_{E} R_{T}\left(y_{0}, \Gamma\right) v\left(\mathrm{~d} y_{0}\right)
$$

We will show that these measures are tight. To this end, consider the sets $\Gamma_{r}:=$ $\left\{y_{0} \in \mathcal{H}^{2} \mid\left\|y_{0}\right\|_{\mathcal{H}^{2}} \leq r\right\} \subset \mathcal{H}^{2} \subset \mathcal{H}^{1}$. Since they are bounded subsets of $\mathcal{H}^{2}$ and the embedding $\mathcal{H}^{2} \subset \mathcal{H}^{1}$ is compact, they are compact subsets of $\mathcal{H}^{1}$. Now, using (40), we show that the measures $R_{T}^{*} \nu$ are concentrated on $\Gamma_{r}$ for sufficiently large $r>0$, hence tight: denoting the complement of a set $A \subset E$ by $A^{c}$ and using Chebychev's 
inequality, we find

$$
\begin{aligned}
R_{T}^{*} v\left(\Gamma_{r}^{c}\right) & =\int_{E} R_{T}\left(y_{0}, \Gamma_{r}^{c}\right) v\left(\mathrm{~d} y_{0}\right)=\int_{E} \frac{1}{T} \int_{0}^{T} \mathbb{E}\left[1_{\Gamma_{r}^{c}}\left(y\left(t ; y_{0}\right)\right)\right] \mathrm{d} t v\left(\mathrm{~d} y_{0}\right) \\
& \leq \int_{E} \frac{1}{T} \int_{0}^{T} \mathbb{E}\left[\frac{\left\|y\left(t ; y_{0}\right)\right\|_{\mathcal{H}^{2}}^{2}}{r^{2}}\right] \mathrm{d} t \nu\left(\mathrm{d} y_{0}\right) \leq \frac{1}{r^{2}} \int_{E} C_{f, H, N, \mathbb{D}} v\left(\mathrm{~d} y_{0}\right) \\
& =\frac{C_{f, H, N, \mathbb{D}}}{r^{2}} .
\end{aligned}
$$

Therefore, the Krylov-Bogoliubov theorem [11, Corollary 3.1.2, p. 22] ensures the existence of an invariant measure.

Remark 4.4 (i) Note that Brzeźniak and Dhariwal [3] prove existence of an invariant measure for a similar system even in the case of the full space, so there might be hope to extend this theorem also in our case.

(ii) The related question of uniqueness of the invariant measure should work in a similar way to [39, Section 5]. For reasons of brevity we do not pursue this here.

Acknowledgements Financial support by the German Research Foundation (DFG) through the IRTG 2235 is gratefully acknowledged. The author would further like to thank Michael Röckner for helpful discussions. Finally, the author is indebted to the anonymous referees for their helpful suggestions that greatly improved the quality of the article.

Funding Open Access funding enabled and organized by Projekt DEAL.

Open Access This article is licensed under a Creative Commons Attribution 4.0 International License, which permits use, sharing, adaptation, distribution and reproduction in any medium or format, as long as you give appropriate credit to the original author(s) and the source, provide a link to the Creative Commons licence, and indicate if changes were made. The images or other third party material in this article are included in the article's Creative Commons licence, unless indicated otherwise in a credit line to the material. If material is not included in the article's Creative Commons licence and your intended use is not permitted by statutory regulation or exceeds the permitted use, you will need to obtain permission directly from the copyright holder. To view a copy of this licence, visit http://creativecommons.org/licenses/by/4.0/.

\section{References}

1. Barbu, V., Da Prato, G.: Existence and ergodicity for the two-dimensional stochastic magnetohydrodynamics equations. Appl. Math. Optim. 56(2), 145-168 (2007)

2. Bessaih, H., Ferrario, B.: Inviscid limit of stochastic damped 2D Navier-Stokes equations. Nonlinearity 27(1), 1-15 (2014)

3. Brzeźniak, Z., Dhariwal, G.: Stochastic tamed Navier-Stokes equations on $\mathbb{R}^{3}$ : the existence and the uniqueness of solutions and the existence of an invariant measure. J. Math. Fluid Mech. 22(2), Paper No. 23, 54 (2020)

4. Brzeźniak, Z., Capiński, M., Flandoli, F.: Stochastic partial differential equations and turbulence. Math. Models Methods Appl. Sci. 1(1), 41-59 (1991)

5. Brzeźniak, Z., Capiński, M., Flandoli, F.: Stochastic Navier-Stokes equations with multiplicative noise. Stochast. Anal. Appl. 10(5), 523-532 (1992)

6. Brzeźniak, Z., Ferrario, B.: Stationary solutions for stochastic damped Navier-Stokes equations in $\mathbb{R}^{d}$. Indiana Univ. Math. J. 68(1), 105-138 (2019) 
7. Cai, X., Jiu, Q.: Weak and strong solutions for the incompressible Navier-Stokes equations with damping. J. Math. Anal. Appl. 343(2), 799-809 (2008)

8. Chueshov, I.D., Millet, A.: Stochastic 2D hydrodynamical type systems: well posedness and large deviations. Appl. Math. Optim. 61(3), 379-420 (2010)

9. Chueshov, I.D., Millet, A.: Stochastic two-dimensional hydrodynamical systems: Wong-Zakai approximation and support theorem. Stoch. Anal. Appl. 29(4), 570-611 (2011)

10. Da Prato, G., Zabczyk, J.: Stochastic Equations in Infinite Dimensions. Encyclopedia of Mathematics and its Applications, vol. 44. Cambridge University Press, Cambridge (1992)

11. Da Prato, G., Zabczyk, J.: Ergodicity for Infinite-Dimensional Systems. London Mathematical Society Lecture Note Series, vol. 229. Cambridge University Press, Cambridge (1996)

12. Dong, Z., Zhang, R.: 3D tamed Navier-Stokes equations driven by multiplicative Lévy noise: existence, uniqueness and large deviations. J. Math. Anal. Appl., 492(1):124404, 48, (2020)

13. Flandoli, F., Gatarek, D.: Martingale and stationary solutions for stochastic Navier-Stokes equations. Probab. Theory Related Fields 102(3), 367-391 (1995)

14. Friz, P.K., Hairer, M.: A Course on Rough Paths. Universitext. Springer, Cham (2014)

15. Gao, H., Liu, H.: Stochastic 3D Navier-Stokes equations with nonlinear damping: martingale solution, strong solution and small time LDP. In: Stochastic PDEs and Modelling of Multiscale Complex System, volume 20 of Interdisciplinary Mathematical Sciences, pp. 9-36. World Scientific Publishing, Hackensack (2019)

16. Gao, H., Liu, H.: Well-posedness and invariant measures for a class of stochastic 3D Navier-Stokes equations with damping driven by jump noise. J. Differ. Equ. 267(10), 5938-5975 (2019)

17. Gawedzki, K., Vergassola, M.: Universality in Turbulence: An Exactly Solvable Model. LowDimensional Models in Statistical Physics and Quantum Field Theory. Springer, Berlin (1996)

18. Gawedzki, K., Vergassola, M.: Phase transition in the passive scalar advection. Physica D Nonlinear Phenomena 138(1), 63-90 (2000)

19. Glatt-Holtz, N., Šverák, V., Vicol, V.: On inviscid limits for the stochastic Navier-Stokes equations and related models. Arch. Ration. Mech. Anal. 217(2), 619-649 (2015)

20. Hofmanová, M., Leahy, J.-M., Nilssen, T.: On the Navier-Stokes equation perturbed by rough transport noise. J. Evol. Equ. 19(1), 203-247 (2019)

21. Huang, J., Shen, T.: Well-posedness and dynamics of the stochastic fractional magneto-hydrodynamic equations. Nonlinear Anal. 133, 102-133 (2016)

22. Kallenberg, O.: Foundations of Modern Probability Probability and its Applications (New York), 2nd edn. Springer, New York (2002)

23. Kraichnan, R.H.: Small-scale structure of a scalar field convected by turbulence. Phys. Fluids 11(5), 945-953 (1968)

24. Liu, H., Gao, H.: Ergodicity and dynamics for the stochastic 3D Navier-Stokes equations with damping. Commun. Math. Sci. 16(1), 97-122 (2018)

25. Liu, H., Lin, L., Sun, C., Xiao, Q.: The exponential behavior and stabilizability of the stochastic 3D Navier-Stokes equations with damping. Rev. Math. Phys. 31(7):1950023, 15 (2019)

26. Liu, W., Röckner, M.: Stochastic Partial Differential Equations: An introduction. Universitext. Springer, Cham (2015)

27. Manna, U., Mohan, M.T.: Two-dimensional magneto-hydrodynamic system with jump processes: well posedness and invariant measures. Commun. Stoch. Anal. 7(1), 153-178 (2013)

28. Manna, U., Mohan, M.T., Sritharan, S.S.: Stochastic non-resistive magnetohydrodynamic system with Lévy noise. Random Oper. Stoch. Equ. 25(3), 155-193 (2017)

29. Marinelli, C., Röckner, M.: On the maximal inequalities of Burkholder, Davis and Gundy. Expo. Math. 34(1), 1-26 (2016)

30. Mikulevicius, R., Rozovskii, B.L.: On equations of stochastic fluid mechanics. In: Stochastics in Finite and Infinite Dimensions, Trends Mathematics, pp. 285-302. Birkhäuser Boston, Boston (2001)

31. Mikulevicius, R., Rozovskii, B.L.: Stochastic Navier-Stokes equations for turbulent flows. SIAM J. Math. Anal. 35(5), 1250-1310 (2004)

32. Mikulevicius, R., Rozovskii, B.L.: Global $L_{2}$-solutions of stochastic Navier-Stokes equations. Ann. Probab. 33(1), 137-176 (2005)

33. Motyl, E.: Stochastic hydrodynamic-type evolution equations driven by Lévy noise in 3D unbounded domains-abstract framework and applications. Stochastic Process. Appl. 124(6), 2052-2097 (2014)

34. Razafimandimby, P.A., Sango, M.: Existence and large time behavior for a stochastic model of modified magnetohydrodynamic equations. Z. Angew. Math. Phys. 66(5), 2197-2235 (2015) 
35. Robinson, J.C., Rodrigo, J.L., Sadowski, W.: The Three-Dimensional Navier-Stokes Equations Cambridge Studies in Advanced Mathematics, vol. 157. Cambridge University Press, Cambridge (2016)

36. Röckner, M., Schmuland, B., Zhang, X.: Yamada-Watanabe theorem for stochastic evolution equations in infinite dimensions. Condens. Matter Phys. 11(2), 247-259 (2008)

37. Röckner, M., Zhang, T.: Stochastic 3D tamed Navier-Stokes equations: existence, uniqueness and small time large deviation principles. J. Differ. Equ. 252(1), 716-744 (2012)

38. Röckner, M., Zhang, T., Zhang, X.: Large deviations for stochastic tamed 3D Navier-Stokes equations. Appl. Math. Optim. 61(2), 267-285 (2010)

39. Röckner, M., Zhang, X.: Stochastic tamed 3D Navier-Stokes equations: existence, uniqueness and ergodicity. Probab. Theory Related Fields 145(1-2), 211-267 (2009)

40. Röckner, M., Zhang, X.: Tamed 3D Navier-Stokes equation: existence, uniqueness and regularity. Infin. Dimens. Anal. Quantum Probab. Relat. Top. 12(4), 525-549 (2009)

41. Schenke, A.: Regularisation and Long-Time Behaviour of Random Systems. PhD thesis, Bielefeld University (2020)

42. Schenke, A.: The tamed MHD equations. J. Evol. Equ. 21(1), 969-1018 (2021)

43. Shen, T., Huang, J.: Ergodicity of stochastic magneto-hydrodynamic equations driven by $\alpha$-stable noise. J. Math. Anal. Appl. 446(1), 746-769 (2017)

44. Sritharan, S.S., Sundar, P.: The stochastic magneto-hydrodynamic system. Infin. Dimens. Anal. Quantum Probab. Relat. Top. 2(2), 241-265 (1999)

45. Sundar, P.: Stochastic magneto-hydrodynamic system perturbed by general noise. Commun. Stoch. Anal. 4(2), 253-269 (2010)

46. Wang, H.: The exponential behavior and stabilizability of the stochastic magnetohydrodynamic equations. Z. Angew. Math. Phys. 69(3), Art. 82, 15 (2018)

47. Wang, S., Li, Y.: Longtime robustness of pullback random attractors for stochastic magnetohydrodynamics equations. Phys. D 382(383), 46-57 (2018)

48. Yamazaki, K.: Global martingale solution to the stochastic nonhomogeneous magnetohydrodynamics system. Adv. Differ. Equ. 21(11-12), 1085-1116 (2016)

49. Yamazaki, K.: Stochastic Lagrangian formulations for damped Navier-Stokes equations and Boussinesq system, with applications. Commun. Stoch. Anal., 12(4):Article 5, 447-471 (2018)

50. Yamazaki, K.: Ergodicity of a Galerkin approximation of three-dimensional magnetohydrodynamics system forced by a degenerate noise. Stochastics 91(1), 114-142 (2019)

51. Yamazaki, K.: Gibbsian dynamics and ergodicity of magnetohydrodynamics and related systems forced by random noise. Stoch. Anal. Appl. 37(3), 412-444 (2019)

52. You, B.: The existence of a random attractor for the three dimensional damped Navier-Stokes equations with additive noise. Stoch. Anal. Appl. 35(4), 691-700 (2017)

53. Zhang, Z., Wu, X., Ming, L.: On the uniqueness of strong solution to the incompressible Navier-Stokes equations with damping. J. Math. Anal. Appl. 377(1), 414-419 (2011)

54. Zhao, W., Li, Y.: Asymptotic behavior of two-dimensional stochastic magneto-hydrodynamics equations with additive noises. J. Math. Phys. 52(7):072701, 18 (2011)

55. Zhou, Y.: Regularity and uniqueness for the 3D incompressible Navier-Stokes equations with damping. Appl. Math. Lett. 25(11), 1822-1825 (2012)

Publisher's Note Springer Nature remains neutral with regard to jurisdictional claims in published maps and institutional affiliations. 